\title{
Progenitor potential of nkx6.1-expressing cells throughout zebrafish life and during beta cell regeneration
}

Aurélie P. Ghaye ${ }^{\dagger}$, David Bergemann ${ }^{\dagger}$, Estefania Tarifeño-Saldivia, Lydie C. Flasse, Virginie Von Berg, Bernard Peers, Marianne L. Voz ${ }^{*+}$ and Isabelle Manfroid ${ }^{* \dagger}$

\begin{abstract}
Background: In contrast to mammals, the zebrafish has the remarkable capacity to regenerate its pancreatic beta cells very efficiently. Understanding the mechanisms of regeneration in the zebrafish and the differences with mammals will be fundamental to discovering molecules able to stimulate the regeneration process in mammals. To identify the pancreatic cells able to give rise to new beta cells in the zebrafish, we generated new transgenic lines allowing the tracing of multipotent pancreatic progenitors and endocrine precursors.

Results: Using novel bacterial artificial chromosome transgenic $n k \times 6.1$ and ascl1 $1 b$ reporter lines, we established that $n k \times 6.1$-positive cells give rise to all the pancreatic cell types and ascl1b-positive cells give rise to all the endocrine cell types in the zebrafish embryo. These two genes are initially co-expressed in the pancreatic primordium and their domains segregate, not as a result of mutual repression, but through the opposite effects of Notch signaling, maintaining $n k \times 6.1$ expression while repressing asc/1b in progenitors. In the adult zebrafish, $n k \times 6.1$ expression persists exclusively in the ductal tree at the tip of which its expression coincides with Notch active signaling in centroacinar/terminal end duct cells. Tracing these cells reveals that they are able to differentiate into other ductal cells and into insulin-expressing cells in normal (non-diabetic) animals. This capacity of ductal cells to generate endocrine cells is supported by the detection of ascl1b in the nkx6.1:GFP ductal cell transcriptome. This transcriptome also reveals, besides actors of the Notch and Wht pathways, several novel markers such as idza. Finally, we show that beta cell ablation in the adult zebrafish triggers proliferation of ductal cells and their differentiation into insulin-expressing cells.

Conclusions: We have shown that, in the zebrafish embryo, $n k x 6.1+$ cells are bona fide multipotent pancreatic progenitors, while asclib + cells represent committed endocrine precursors. In contrast to the mouse, pancreatic progenitor markers $n k x 6.1$ and $p d x 1$ continue to be expressed in adult ductal cells, a subset of which we show are still able to proliferate and undergo ductal and endocrine differentiation, providing robust evidence of the existence of pancreatic progenitor/stem cells in the adult zebrafish. Our findings support the hypothesis that nkx6.1+ pancreatic progenitors contribute to beta cell regeneration. Further characterization of these cells will open up new perspectives for anti-diabetic therapies.
\end{abstract}

Keywords: $n k \times 6.1$, ascl1, pancreas, duct, centroacinar cells, beta cells, stem cells, lineage tracing, multipotent progenitors, regeneration, diabetes, zebrafish, Notch, Wnt

\footnotetext{
* Correspondence: mvoz@ulg.ac.be; isabelle.manfroid@ulg.ac.be

'Equal contributors

Laboratory of Zebrafish Development and Disease Models (ZDDM),

GIGA-Research, (Sart-Tilman) University of Liège, Avenue de l'Hôpital 1, B34,

4000 Liège, Belgium
}

\section{Biomed Central}

(C) 2015 Ghaye et al. Open Access This article is distributed under the terms of the Creative Commons Attribution 4.0 International License (http://creativecommons.org/licenses/by/4.0/), which permits unrestricted use, distribution, and reproduction in any medium, provided you give appropriate credit to the original author(s) and the source, provide a link to the Creative Commons license, and indicate if changes were made. The Creative Commons Public Domain Dedication waiver (http://creativecommons.org/publicdomain/zero/1.0/) applies to the data made available in this article, unless otherwise stated. 


\section{Background}

The pancreas is composed of an exocrine compartment with acinar and ductal cells, which secrete and transport digestive enzymes into the gut, and an endocrine compartment, which regulates glucose homeostasis by secreting pancreatic hormones into the bloodstream. Loss of pancreatic insulin-producing cells (beta cells) is a hallmark of diabetes. An attractive therapeutic approach to cure this disease is to stimulate beta cell regeneration in vivo from another pancreatic cell type or from progenitors. However, the regenerative capacity of beta cells is very limited in mammals and the cellular and molecular mechanisms involved need to be well understood before we will be able to stimulate this process. The zebrafish (Danio rerio), owing to its phenomenal capacity to restore beta cells after targeted cell ablation [1-3], has become an attractive model organism for the study of the regeneration process. To this end, tools need to be developed, especially to define the source of the new beta cells. So far, no zebrafish transgenic lines have been available to allow a lineage tracing of either multipotent pancreatic progenitors, giving rise to both exocrine and endocrine tissues, or pancreatic endocrine precursor cells.

The pancreas develops from two domains, called the dorsal bud and the ventral bud, which emerge from the foregut endoderm $[4,5]$. In zebrafish, the dorsal bud generates the first wave of endocrine cells, which cluster at 24 hours post fertilization (hpf) to form the principal islet [6]. The ventral bud emerges anteriorly to the dorsal bud at $32 \mathrm{hpf}$ and gives rise to acinar, ductal, and to a second wave of endocrine cells $[4,7,8]$. These late endocrine cells originate either from the extrapancreatic ducts (EPDs) and contribute to the expansion of the principal islet [9-11] or from the intra-pancreatic ducts (IPDs) and form small secondary islets all along them. These IPDs contain pancreatic Notch-responsive cells (PNCs), which represent a population of progenitors of endocrine cells and ductal cells but not of acinar cells $[7,12]$.

Notch signaling pathway controls the differentiation of pancreatic cells both in zebrafish and mice (reviewed by [13]). One of its functions is to maintain a pool of progenitors in an undifferentiated state through the repression of genes of the achaete scute-like (ASCL) family or of the atonal-related protein (ARP) family. In the murine pancreas, Notch signaling prevents endocrine cell differentiation through the repression of neurog3 [14]. In zebrafish, neurog3 is not expressed in the pancreas and therefore the control of endocrine cell fate is fulfilled by other ASCL/ARP factors, namely Ascl1b and Neurod1, which are both repressed by Notch signaling [15]. Exactly like the inactivation of murine Neurog3, their simultaneous inactivation completely prevents endocrine cell differentiation leading to the loss of all hormone-secreting cells [15]. ascl1b is the earliest pancreatic marker identified during zebrafish development, its expression starting at the end of gastrulation in the prospective pancreatic region (10 hpf). ascl1b is transiently expressed during the formation of the dorsal bud (10-17 hpf) and, like murine Neurog3, is not detected in hormone-expressing cells. Later, in the ventral bud, ascl1b expression is turned on when the endocrine cell differentiation program is induced through the blocking of Notch signaling [7, 12, 16]. This Notch inactivation triggers a massive expression of ascl1b in IPDs [15]. These data suggest that ascl1b expression is restricted to the committed endocrine precursors. However, the observation that the onset of ascl1b expression in the prospective pancreatic region precedes all other known pancreatic progenitor markers raises the possibility of the multipotency of the first $a s c l 1 b+$ cells.

Another key factor for pancreatic development is the homeobox transcription factor Nkx6.1. In the mouse, it is expressed in the multipotent progenitors during early pancreatic development [17], and, in the zebrafish, $n k x 6.1$ is expressed early in the pancreatic primordium of the dorsal bud (from $11.5 \mathrm{hpf}$ onwards) [18]. At later developmental stages in the mouse embryo, $N k x 6.1$ becomes restricted to the endocrine/duct bipotential trunk domain [19]. Similarly, $n k x 6.1$ is first broadly expressed in the zebrafish pancreatic ventral bud primordium [20], then segregates from the ptf1a+ acinar cells to persist in the primitive ducts [20-22] that will give rise to the mature ducts and to secondary islets [7]. In the mouse, $N k x 6.1$ is expressed in the differentiated beta cells [23] while in the zebrafish, $n k x 6.1$ is never expressed in beta cells nor in the other pancreatic hormoneexpressing cells [18]. These data suggest that in zebrafish $n k x 6.1$ also marks multipotent pancreatic progenitors. However, previous findings suggested that the early ventral bud primordium was composed of a heterogeneous population of pancreatic cells comprising Notchresponsive cells, giving rise to ductal and endocrine cells, separated from the $p t f 1 a+$ cells, which generate the acinar cells [7]. This study raises the question of the identity of the multipotent pancreatic progenitors in the zebrafish ventral pancreatic bud and its derivatives.

Here, we show that $n k x 6.1$ labels multipotent pancreatic progenitors giving rise to all of the different pancreatic cell types (endocrine, ductal, and acinar) while ascl1b marks endocrine precursors leading to the different endocrine cell types. For this purpose, we have generated two novel bacterial artificial chromosome (BAC) transgenic $n k x 6.1$ and ascl1b reporter lines, $\operatorname{Tg}(n k x 6.1: e G F P)$ and $\operatorname{Tg}\left(\right.$ ascl1b:eGFP-2A-creER ${ }^{T 2}$ ), that both faithfully recapitulate the expression of the $n k x 6.1$ and ascl1b endogenous genes. Using these novel transgenic 
tools, we were able to analyze in detail the interdependency between these two factors and their relationship with the Notch signaling pathway. We also demonstrate that $n k x 6.1$ expression persists in the adult ductal tree, notably in the centroacinar/terminal end duct cells (CACs), for which we show that they are able to differentiate into insulinexpressing cells in vivo. By isolating $n k x 6.1: \mathrm{eGFP}+$ cells from the dissected pancreases of adult fish, we determined the transcriptome of adult pancreatic ductal cells, which revealed the expression of several regulatory genes potentially involved in endocrine regeneration. Finally, we provide evidence that regenerating beta cells also originate from ductal cells.

\section{Results}

The bacterial artificial chromosome reporter $T g$ (nkx6.1:eGFP) recapitulates in vivo the expression of the endogenous $n k x 6.1$ gene

To label the $n k x 6.1$-expressing cells, we generated a transgenic line driving the expression of the enhanced green fluorescent protein (eGFP) under the control of $n k x 6.1$ regulatory regions. We engineered a BAC spanning from $55 \mathrm{~kb}$ upstream to $95 \mathrm{~kb}$ downstream of the $n k x 6.1$ gene and inserted the eGFP coding regions into exon 1, replacing the beginning of the $n k x 6.1$ open reading frame (Additional file 1: Fig. S1A). This BAC reporter construct was introduced into the zebrafish genome using the Tol2 transposon system $[24,25]$ and the stable transgenic line $\operatorname{Tg}(n k x 6.1: e G F P)$ obtained showed expression of green fluorescent protein (GFP) in the nervous system and in the pancreas, which mirrors the endogenous Nkx6.1 protein expression (Additional file 1: Fig. S1B). Detailed comparison of the localization of these two proteins in the pancreas during development confirmed that GFP is indeed co-expressed with Nkx6.1 (Fig. 1). Indeed, together with the endogenous Nkx6.1 protein [18], GFP is expressed at the base of the endocrine islet at 24 and $30 \mathrm{hpf}$ (Fig. 1b, c), in the ventral bud at 38 and 48 hpf (Fig. 1d, e), and in IPDs and EPDs at 4 days post fertilization (dpf) (Fig. 1f, f). In contrast, at earlier stages, GFP was detected in a subset of Nkx6.1+ cells, probably due to the delay of GFP expression compared to Nkx6.1. Indeed, at $17 \mathrm{hpf}$, about $75 \%$ of the Nkx6.1+ cells showed detectable GFP expression (Fig. 1a, a') and at $14 \mathrm{hpf}$, this proportion dropped even further to about 25-30 \% (data not shown). Conversely, a few hours after the onset of $n k x 6.1$ gene expression, some GFP+/Nkx6.1- cells were also detected (green arrows, Fig. 1b'-e'). This GFP labeling is not the result of an ectopic expression of the $g f p$ transcript, as double fluorescent whole-mount in situ hybridization (WISH) showed that the $g f p$ transcripts are present in the same pancreatic domain as $n k x 6.1$ transcripts (data not shown) and importantly, like $n k x 6.1, g f p$ transcripts were not found in hormoneexpressing cells (Additional file 1: Fig. S1C-C", D-D"). Hence, prolonged GFP detection is rather due to the well-known high stability of GFP $( \pm 24 \mathrm{~h}$ half-life [26]), which persists in cells where Nkx6.1 protein is no longer found. This is nicely illustrated at $30 \mathrm{hpf}$, where strong GFP expression is detected at the base of the forming islet where Nkx6.1+ pancreatic progenitors are located, while weak GFP labeling is found dorsally within the islet, where differentiated endocrine cells, devoid of Nkx6.1, are clustered (Fig. 1c). As expected, this prolonged GFP detection will gradually fade away, finally to disappear completely in the differentiated endocrine cells (Fig. 1f).

The high stability of GFP allowed us to perform shortterm lineage tracing to follow the immediate progeny arising from $n k x 6.1+$ cells.

\section{nkx6.1-expressing cells are multipotent progenitors giving rise to all pancreatic cell lineages}

Using short-term lineage tracing, we first assessed if $n k x 6.1+$ cells can give rise to the first wave of endocrine cells of the dorsal bud using the Isl1 marker, which labels all mature endocrine cells. In contrast to the endogenous $n k x 6.1$ and the $n k x 6.1: G F P$ transcripts, which are not co-expressed with isl1 ([18] and Additional file 1: Fig. S1D-D"), GFP was detected in $40 \pm 3.8 \%$ of Isl1+ cells in $\operatorname{Tg}(n k x 6.1: e G F P)$ embryos $(n=10)$ at $30 \mathrm{hpf}$ indicating that $n k x 6.1$ cells can give rise to endocrine cells (Fig. 2a-a"). Also, we found GFP in all different endocrine cell types, i.e. in $35 \pm 18.8 \%$ of insulin+ (Ins+) cells $(n=21)$ (Fig. $2 b-b "), 42 \pm 12.5 \%$ somatostatin + (Sst + ) cells $(n=4)$ (Fig. $\left.2 \mathrm{c}-\mathrm{c}^{\prime \prime}\right)$, and $77 \pm 3.7 \%$ of glucagon + $(\mathrm{Gcg}+)$ cells $(n=5)$ (Fig. $2 \mathrm{~d}-\mathrm{d} ")$. The percentage of GFP+ cells in the different endocrine subtypes appeared to depend on the onset of expression for each hormone, which is from $15 \mathrm{hpf}$ onward for ins, $17 \mathrm{hpf}$ for sst2 and $21 \mathrm{hpf}$ for gcga. Therefore, when the first hormone-expressing cells differentiate from the pool of $n k x 6.1+$ progenitors, only a minority of them have accumulated enough GFP to be detected, as explained above (see Fig. 1a and data not shown).

Next, we analyzed whether the $n k x 6.1+$ cells also contribute to the cells originating from the ventral bud (ductal, acinar, and secondary islets). With Nkx6.1 being expressed in all pancreatic ductal cells (as shown in Fig. 1e), we detected accordingly an expression of GFP in all pancreatic ducts labeled by $2 \mathrm{~F} 11$ antibody (Fig. 2f, f). In contrast, while endogenous $n k x 6.1$ never co-localizes with trypsin (data not shown), a marker of mature acinar cells, GFP was detected in a large majority of acinar cells at 55 hpf $(70 \pm 25 \%$ of trypsin + cells $(n=8))$, the stage when acinar cells have just begun to differentiate (Fig. 2e, e'). Here again, the prolonged GFP 

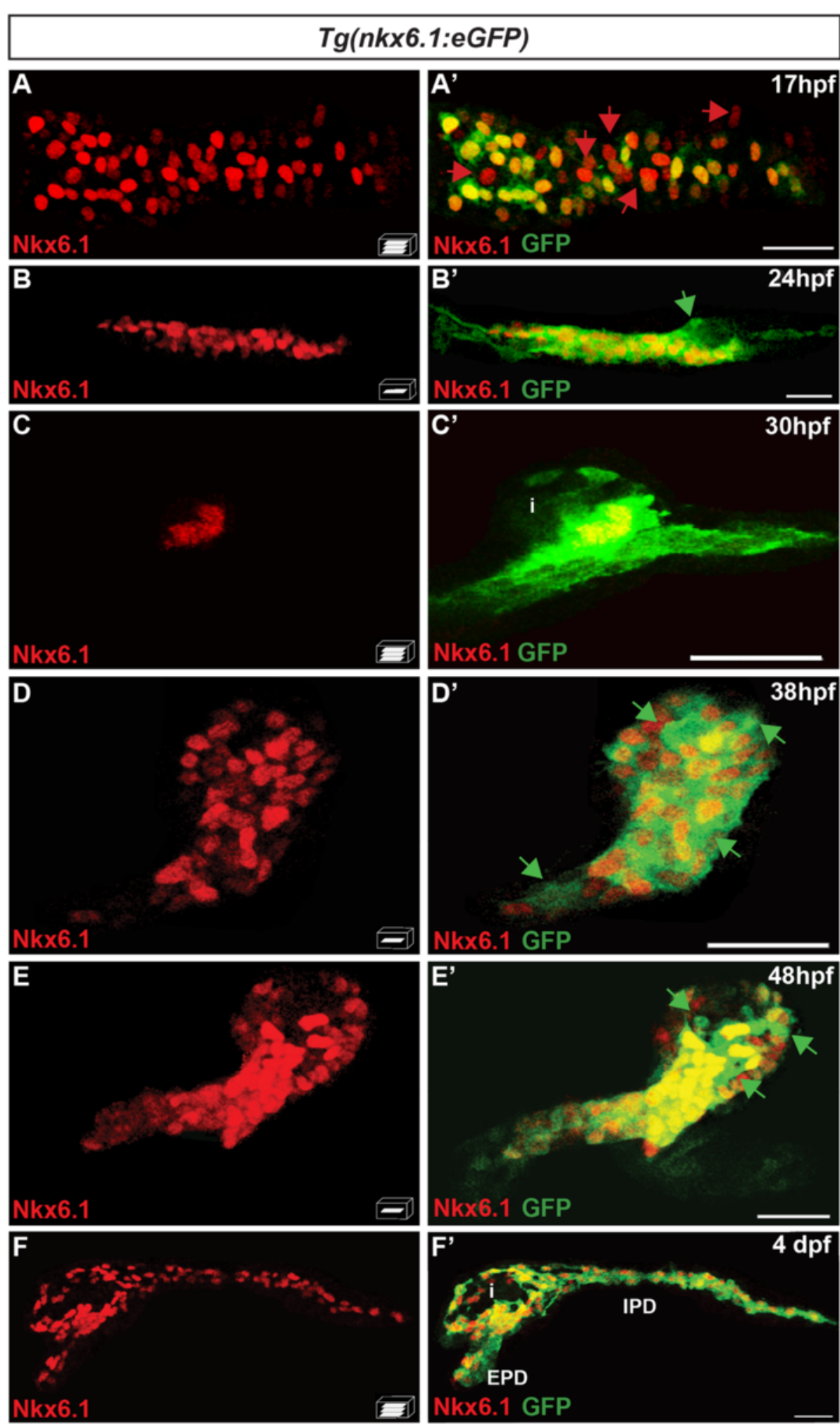

Fig. 1 The bacterial artificial chromosome reporter line $\operatorname{Tg}(n k \times 6.1:$ eGFP) mirrors the expression of the endogenous $n k \times 6.1$ gene. Immunodetection of endogenous Nkx6.1 (red) and GFP (green) in Tg(nkx6.1:eGFP) embryos of the indicated stages. Green arrows point to Nkx6.1-/GFP+ cells and red arrows to Nkx6.1+/GFP- cells. All views are either lateral (b, $\mathbf{b}^{\prime}, \mathbf{c}$, and $\left.\mathbf{c}^{\prime}\right)$ or ventral $\left(\mathbf{a}, \mathbf{a}^{\prime}, \mathbf{d}, \mathbf{d}^{\prime}, \mathbf{f}\right.$, and $\left.\mathbf{f}^{\prime}\right)$ with the anterior part to the left. They represent either z-plane confocal images $(\mathbf{b}, \mathbf{d}, \mathbf{e})$ or confocal projection images $(\mathbf{a}, \mathbf{c}, \mathbf{f})$. Scale bars $=30 \mu \mathrm{m}$. EPD extra-pancreatic duct, IPD intra-pancreatic duct, $i$ islet 

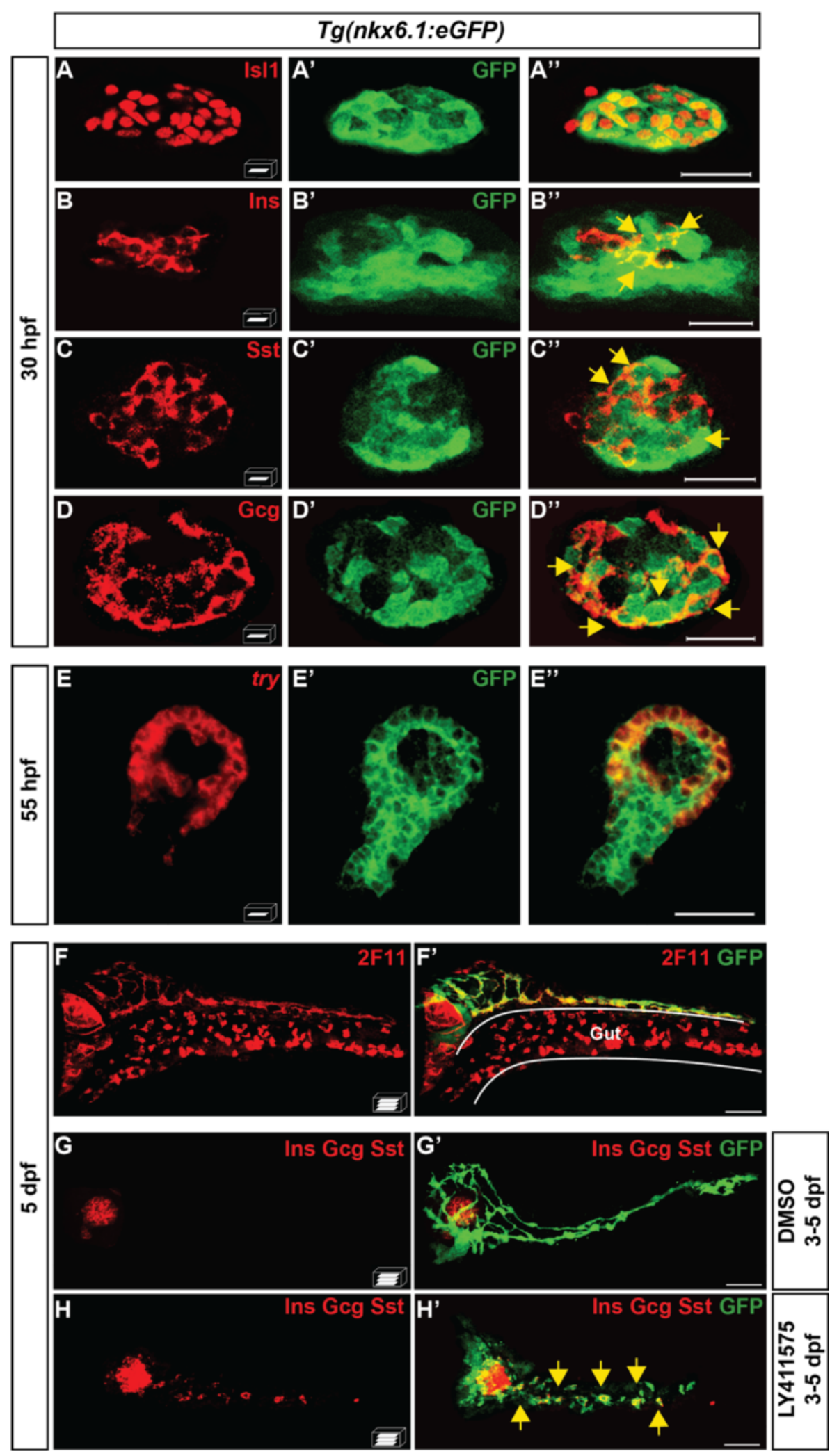

Fig. 2 (See legend on next page.) 
(See figure on previous page.)

Fig. 2 nkx6.1-expressing cells give rise to all pancreatic cell types. a-d" Immunodetection in 30-hpf $\operatorname{Tg}(n k x 6.1: e G F P)$ embryos of GFP with Isl1 (a-a"), Ins (b-b"), Sst (c-c"), or Gcg (d-d"). Yellow arrows point to cells co-expressing GFP and the respective hormones. e-e" Fluorescent whole-mount in situ hybridization (WISH) of 55-hpf Tg(nkx6.1:eGFP) embryos using a try probe followed by GFP immunodetection. $\mathbf{f}, \mathbf{f}^{\prime}$ Immunodetection in 5-dpf Tg(nkx6.1:eGFP) embryos of GFP and of the hepato-pancreatic duct marker 2F11. g-h' Immunodetection of GFP and of Ins, Gcg, and Sst hormones in 5-dpf Tg(nkx6.1:eGFP) embryos treated from 3 to $5 \mathrm{dpf}$ with dimethyl sulfoxide (DMSO) (g) or with the Notch signaling inhibitor, LY411575 (h). Yellow arrows point to secondary endocrine GFP+/hormones+ cells found in IPDs. All views are ventral with the anterior part to the left and represent either z-plane confocal images (a-e) or confocal projection images (f-h). Scale bars $=20 \mu \mathrm{m}(\mathbf{a}-\mathbf{e})$ or $40 \mu \mathrm{m}(\mathbf{f}-\mathbf{h})$

detection in the acinar cells gradually disappears and, from $3 \mathrm{dpf}$, the acinar cells are no longer labeled with GFP (shown at 5 dpf in Fig. 2f'). And finally, to determine whether $n k x 6.1+$ cells can give rise to the secondary islets, emerging from IPDs, we treated $\operatorname{Tg}(n k x 6.1: e G F P)$ larvae with the Notch-signaling inhibitor LY411575 from 3 to $5 \mathrm{dpf}$ to increase the number of late endocrine cells and thereby facilitate their detection $[7,12,16]$. In LY411575-treated larvae, we observed an increase of the endocrine cells in the principal islet and the appearance of numerous endocrine cells in the pancreatic tail (yellow arrows, Fig. 2h, h'), as previously reported. All these endocrine cells are colabeled with GFP $(n=4)$ indicating that the $n k x 6.1+$ ductal cells can also give rise to the secondary islets.

These data indicate that $n k x 6.1$-expressing cells are multi-lineage pancreatic progenitors, which can differentiate into endocrine, acinar, and ductal cells.

\section{ascl1 b-expressing cells give rise exclusively to the endocrine lineage}

To determine whether ascl1b is expressed in the multipotent pancreatic progenitors or in the endocrine precursors, we determined the cell fate of the ascl1b-expressing cells. To that end, a BAC reporter $\operatorname{Tg}$ (ascl1b:eGFP-2A-creER ${ }^{T 2}$ ) was engineered where the bicistronic transcript eGFP$2 A$-creER $R^{T 2}$ is under the control of the promoter and regulatory sequences of $a s c l 1 b$. Thus, we replaced the beginning of the ascl1b open reading frame with an $e G F P-2 A$-creER $R^{T 2}$ cassette (Additional file 2: Fig. S2A). The expression profile of GFP in the stable transgenic line $\operatorname{Tg}\left(\right.$ ascl1b:eGFP-2A-creER $\left.{ }^{T 2}\right)$ faithfully recapitulates the expression of the endogenous ascl1b transcript (Additional file 2: Fig. S2B-D).

Cell fate experiments were performed with Cre/loxPbased lineage tracing approaches by crossing the $T g$ (ascl1b:eGFP-2A-creER ${ }^{T 2}$ ) with Cre-responder transgenic lines, either $\operatorname{Tg}$ (ubi:loxP-AmCyan-loxP-ZsYellow), termed $\operatorname{Tg}(u b i: C S Y)$ [27], or Tg(ubi:loxP-eGFP-LoxP$m$ Cherry), termed $\operatorname{Tg}$ (ubi:Switch) [28] (Fig. 3a). The double-transgenic embryos were treated five times with 4-hydroxytamoxifen (4OHT) from 11 to $15 \mathrm{hpf}$, the period when ascl1b expression reaches its maximal level [15], and the embryos were analyzed at 48 or 72 hpf. With these five $4 \mathrm{OHT}$ treatments, many asclib- expressing cells have undergone CRE recombination while no recombination was detected in the treated single-transgenic embryos used as control (data not shown). The CRE-mediated recombination (rec) marker (standing for either ZsYellow or mCherry) analyzed in double-transgenic embryos at $48 \mathrm{hpf}$ was detected in $38 \pm 4.3 \%$ of Isl $1+$ cells $(n=5)$, indicating that $a s c l 1 b+$ cells give rise to the endocrine cells of the dorsal bud (Fig. 3b-b"). In a similar way, the rec marker was detected in $58 \pm 7.1 \%$ of the Ins+ cells $(n=9)$ (Fig. 3c-c") and in $59 \pm 3.7 \%$ of the Gcg+ cells $(n=9)$ (Fig. $3 d-d ")$. In contrast, the rec marker, clearly visible in the endocrine islet, was not detected at $72 \mathrm{hpf}$ in the ductal cells, labeled by Nkx6.1, nor in the acinar cells, which surround them (Fig. 3e, e'), indicating that $a s c l 1 b+$ cells cannot give rise to exocrine cells. Finally, we determined whether the $a s c l 1 b+$ cells give rise to the secondary islets emerging from IPDs, either artificially induced by inhibiting the Notch pathway or naturally occurring in 20-dpf larvae. As the combined treatment of LY411575 with $4 \mathrm{OHT}$ was lethal, we performed short-term lineage tracing based on GFP expression (instead of Cre/loxP-based lineage tracing analyses). As shown in Fig. 3f, g, LY411575 treatment from 3 to 5 days of the $T g$ (ascl1b:eGFP-2A-creER ${ }^{T 2}$ ) larvae led to the appearance of GFP cells all along the IPDs, most of these cells being also positive for glucagon or insulin hormones (yellow arrows, Fig. 3g, g'), indicating that $a s c l 1 b+$ cells give rise to induced secondary islets. To trace the naturally occurring endocrine cells, we treated $\operatorname{Tg}$ (ascl1b:eGFP-2A-creER ${ }^{T 2}$ ); $\operatorname{Tg}$ (ubi:Switch) larvae with $4 \mathrm{OHT}$ at 13,14 , and $17 \mathrm{dpf}$ and analyzed the larvae at 20 dpf. The rec marker was detected within the principal islet (Fig. 3h, h') as well as in secondary islets (Fig. 3i, i') confirming that $a s c l 1 b+$ cells can give rise to secondary islets.

In conclusion, our data demonstrate that $a s c l 1 b+$ cells exclusively give rise to the endocrine cells originating from both the dorsal and ventral buds.

\section{$n k x 6.1$ and ascl1b are first co-expressed in the endocrine precursors of the dorsal bud but rapidly their expression domain segregates}

As presented above, $n k x 6.1$ is expressed in the multipotent pancreatic progenitors and $a s c l 1 b$ in the endocrine precursors; we therefore analyzed the relationship between these two populations by comparing the Nkx6.1 


$$
\text { A }
$$

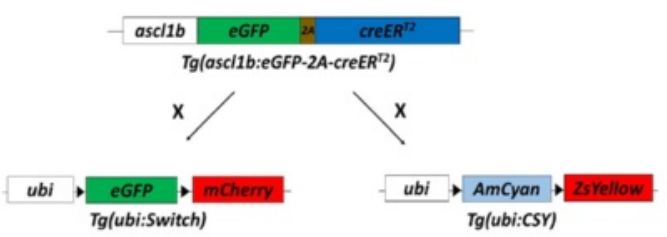

\section{$T g$ (ascl1b:eGFP-2A-creER $\left.{ }^{T 2}\right) 4 \mathrm{OH}$ at $11,12,13,14$ and $15 \mathrm{hpf}$}
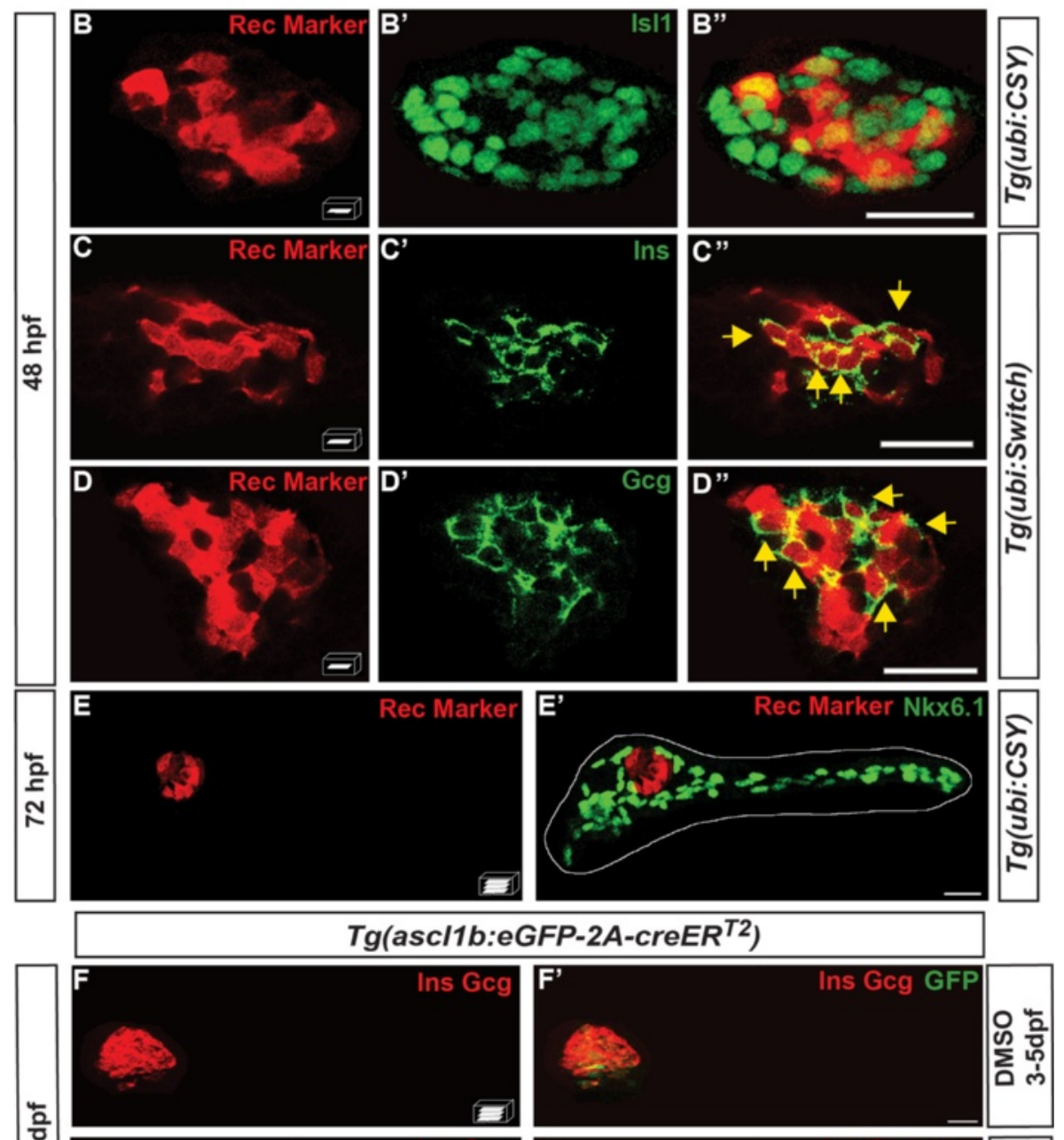

in
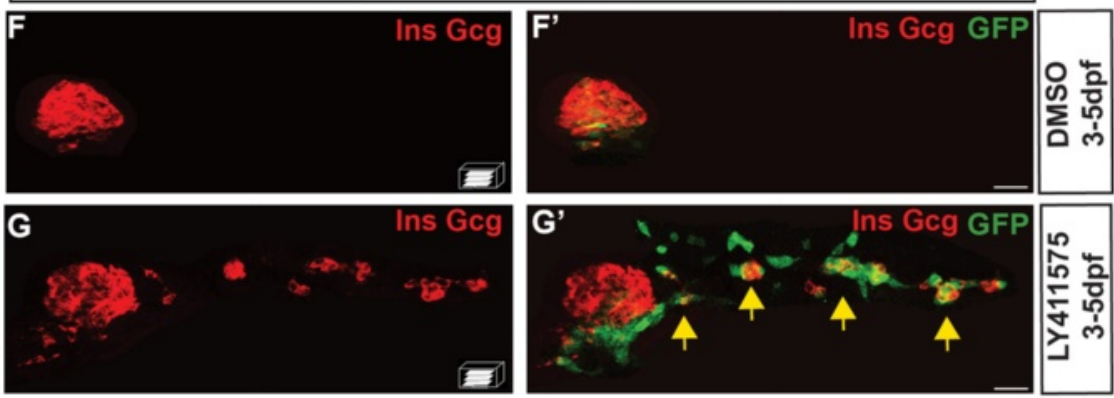

$T g$ (ascl1b:eGFP-2A-creER ${ }^{T 2}$ ) $\quad 40 \mathrm{OH}$ at 13, 14 and $17 \mathrm{dpf}$
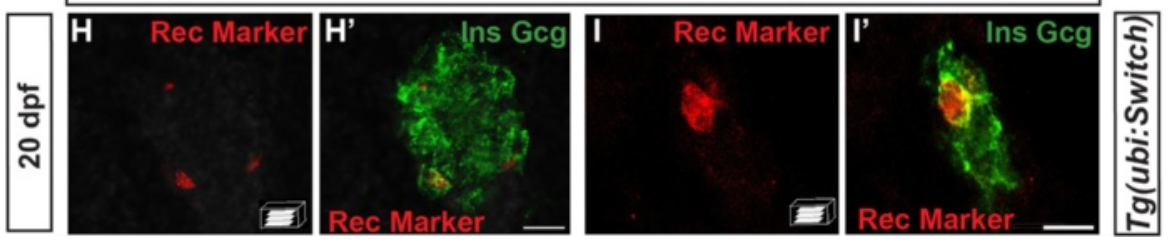

Fig. 3 (See legend on next page.) 
(See figure on previous page.)

Fig. 3 asclib-expressing cells give rise exclusively to endocrine cells of both dorsal and ventral bud. a-e', $\mathbf{h}$-i' Genetic lineage tracing using the Cre-loxP system. a Schematic representation of the genetic lineage tracing experiments. The transgenic line Tg(asclib:eGFP-creER ${ }^{T 2}$ ) was crossed with the Tg(ubi:loxP-eGFP-loxP-mCherry) line, abbreviated Tg(ubi:Switch), or with the Tg(ubi:loxP-AmCyan-loxP-ZsYellow) line, abbreviated Tg(ubi:CSY), treated with 4-hydroxytamoxifen (4OHT) at 11, 12, 13, 14, and 15 hpf (b-e') or at 13, 14, and $17 \mathrm{dpf}(\mathbf{h}$-i') and fixed for analysis at the indicated times. Black triangles in a represent loxP sites. b-e' Immunodetection of CRE-mediated rec markers (ZsYellow or mCherry, red) and Isl1 (b-b"), Ins $\left(\mathbf{c}-\mathbf{c}^{\prime \prime}\right)$, Gcg $(\mathbf{d}-\mathbf{d}$ "), or Nkx6.1 (e, e') in 4OHT-treated embryos (green). The dotted white line delimits the pancreas (e'). Yellow arrows point to cells co-expressing rec marker (ZsYellow or mCherry) and the respective hormones (Ins or Gcg). $\mathbf{f}-\mathbf{g}$ ' Short-term lineage tracing: immunodetection of GFP and the Ins and Gcg hormones in 5-dpf Tg(asclib:eGFP-creER ${ }^{T 2}$ ) embryos treated from 3 to $5 \mathrm{dpf}$ with DMSO (f, f') or with the Notch signaling inhibitor, LY411575 (g, g'). Yellow arrows in $\mathbf{g}^{\prime}$ point to GFP+/Inst/Gcg+ secondary endocrine cells found in the IPDs. $\mathbf{h}-\mathbf{i}^{\prime}$ Immunodetection at $20 \mathrm{dpf}$ of the CRE-mediated rec marker mCherry together with Ins and $\mathrm{Gcg}$ in $4 \mathrm{OHT}$-treated larvae. All views are ventral with the anterior part to the left and represent z-plane confocal images (b-d") or confocal projection images (e-i'). Scale bars $=20 \mu \mathrm{m}$

and GFP proteins in $\operatorname{Tg}\left(\right.$ ascl1b:eGFP-2A-creER $\left.{ }^{T 2}\right)$ embryos. At $14 \mathrm{hpf}$, the ascl1b:eGFP cells delineate two lines adjacent to the midline (Fig. 4a-a"). These cells correspond to the most medial endodermal cells (indicated by $M$ in Fig. 4a'), reported to give rise mostly to pancreatic endocrine cells $[29,30]$. At this stage, all of these ascl1b:eGFP cells also express Nkx6.1. In contrast, the Nkx6.1 expression domain is larger and, in addition to its expression in the hypochord (indicated by $H$ in Fig. 4a), it is also expressed in the lateral cells, reported to give rise to exocrine and intestinal cells [29]. Rapidly, these two domains segregate since, as early as 1 hour later, the majority of ascl1b:eGFP+ cells no longer express Nkx6.1 (Fig. 4b-b"). Thus, separation of the two domains is largely completed when hormone-expressing cells start differentiating.

To determine whether such segregation results from a mutual repression, we examined whether the loss of ascl1b leads to an increase of $n k x 6.1$ expression and vice versa. We generated $a s c l 1 b$ and $n k x 6.1$ loss-of-function mutants using the CRISPR/cas9 genome editing technology [31] (see "Methods" and Additional file 3: Fig. S3A, A' and Additional file 4: Fig. S4A, A'). As shown in Additional file 3: Fig. S3, the loss of ascl1b does not affect the expression of $n k x 6.1$ (Additional file 3: Fig. S3D, E) while it significantly reduces the number of $s s t 2+$ cells (Additional file 3: Fig. S3B, C), as reported for the $a s c l 1 b$ morphants [15]. In the same way, ascl1b expression does not increase in $n k x 6.1$ loss-of-function mutant embryos (Additional file 4: Fig. S4F, G), for which the effective loss of $n k x 6.1$ expression was confirmed by immunodetection of Nkx6.1 (Additional file 4: Fig. S4B, C) and by the drastic reduction in the number of Gcg+ cells (Additional file 4: Fig. S4D, E), as reported for the $n k x 6.1$ morphants [18].

\section{ascl1b and nkx6.1 are regulated in an opposite way by the Notch signaling pathway}

We then tested whether the segregation of the asclib and $n k x 6.1$ expression domains results from a different response to the Delta/Notch signaling pathway. To analyze the impact of Notch signaling on $n k x 6.1$ and ascl1b expression, we performed Notch loss- and gainof- function analyses. We first analyzed the expression

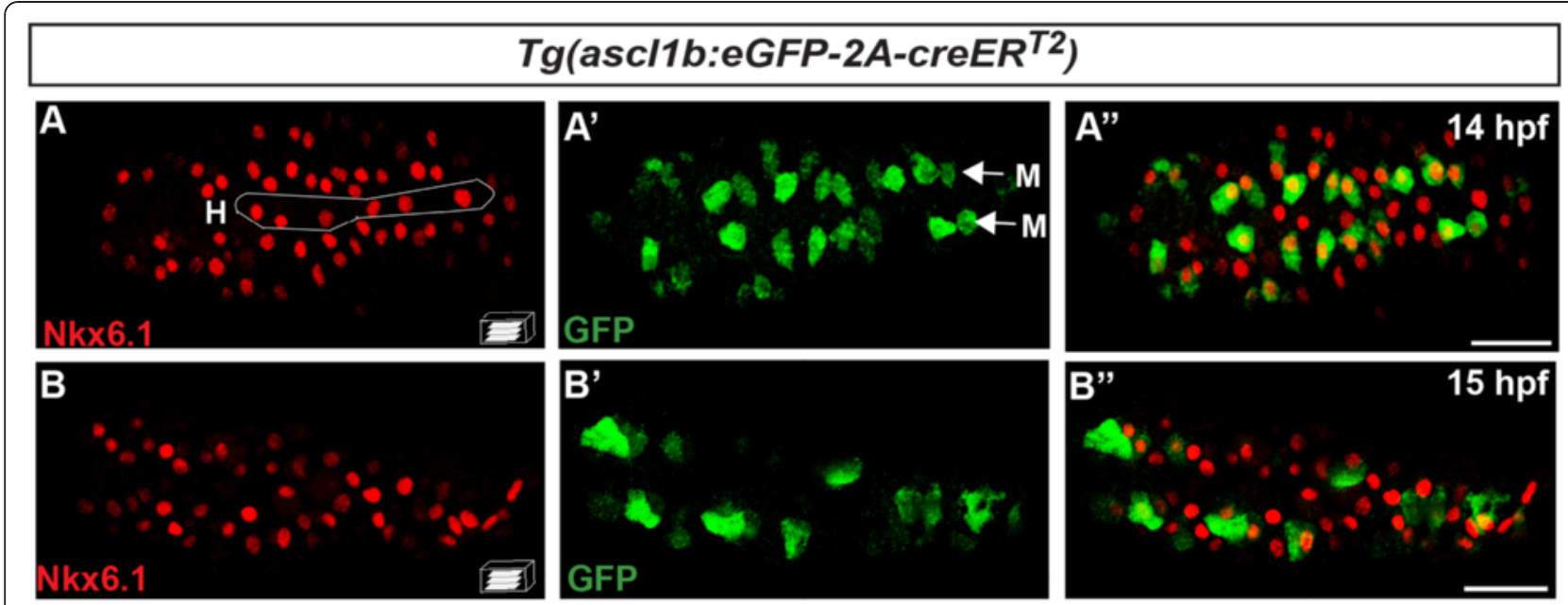

Fig. $4 \mathrm{nkx6.1}$ and ascl1b are first co-expressed in the endocrine precursors of the dorsal bud but rapidly their expression domain moves apart. Immunodetection of endogenous Nkx6.1 and GFP in Tg(ascl1b:eGFP-creER T2) embryos at 14 hpf (a-a") and 15 hpf (b-b"). All views are ventral with the anterior part to the left and represent confocal projection images. Scale bars $=40 \mu \mathrm{m}$. $\mathrm{H}$ hypochord, $\mathrm{M}$ medial cells 
of $n k x 6.1$ and $a s c l 1 b$ in mind bomb mutants (mib ${ }^{t a 52 b}$ ) in which Notch signaling is disrupted [32]. As previously reported [15], expression of ascl1b is strongly increased in mib embryos at $15 \mathrm{hpf}$ (Fig. 5a, b), in both the pancreas and the nervous system. The opposite effect was observed for $n k x 6.1$ whose expression is reduced at the same stage (Fig. 5c, d) and completely lost at $18 \mathrm{hpf}$ (Fig. 5e, f). This suggests that the Notch signaling pathway is essential for maintaining $n k x 6.1$ expression but not for its initiation. This was confirmed by the finding that, at $13 \mathrm{hpf}, n k x 6.1$ expression was unchanged in mib mutants while ascl $1 b$ was already upregulated (Additional file 5: Fig. S5A-D).

The same conclusion was drawn for the ventral bud where treatment for 3-5 dpf with the LY411575 Notch inhibitor led to a complete loss of Nkx6.1 expression and a drastic increase of ascl1b:eGFP at 5 dpf (Fig. 5g, h). Like the dorsal bud, the initiation of $n k x 6.1$ expression in the ventral bud is not dependent on Notch signaling as its expression at 34 hpf was not perturbed in the mib mutant (Additional file 5: Fig. S5E, F).

For gain-of-function approaches, we crossed the $\operatorname{Tg}$ (hsp70:Gal4) with $\operatorname{Tg}($ UAS:NICD) [33] and heatshocked the embryos at $11 \mathrm{hpf}$ to overexpress the Notch intracellular domain (NICD). At 3 hours after the heatshock, we observed a complete loss of ascl1b expression (Fig. 5i, j) concomitant with an increase of $n k x 6.1$ in the NICD overexpressed embryos (Fig. 5k, l). This increase was even more important at $30 \mathrm{hpf}$, when a drastic expansion in the number of $n k x 6.1+$ cells was observed in the embryos overexpressing NICD (Fig. 5n) compared to the control (Fig. 5m).

In conclusion, these data show that Notch signaling represses ascl1b expression while it is essential for maintaining $n k x 6.1$ expression. By contrast, the initiation of $n k x 6.1$ expression is independent of Notch activity, both in the dorsal and the ventral buds.

Most, but not all, Nkx6.1+ cells are Notch-responsive cells $n k x 6.1$ being dependent on Notch signaling for maintaining its own expression, this prompted us to compare the location of the Notch-responsive cells and the Nkx6.1+ cells using the $\operatorname{Tg}(\operatorname{Tp} 1:$ VenusPest $)$ [16] or $\operatorname{Tg}(T p 1: e G F P)$ [12] lines in which fluorescent markers are under the control of Notch-responsive elements (Tp1). In the prospective dorsal bud, we could detect Venus labeling in a subset of Nkx6.1 cells at $14 \mathrm{hpf}$ (Fig. 6a, a') while 3 hours later (17 hpf), Venus was found in the vast majority of Nkx6.1+ cells (Fig. 6b, b'). Similarly, at the beginning of the formation of the ventral bud (38 hpf), only a subpopulation of Nkx6.1+ cells present some Notch activity (Fig. 6c, c') while at 65 hpf, the vast majority of Nkx6.1+ cells show Notch activity with the exception of the EPD anlagen (Fig. 6d, d'), known to be Notch inactive [34]. At 4 days, virtually all Nkx6.1+ cells are Notch-responsive in the IPDs (but the EPDs are still devoid of Notch activity) (Fig. 6e, e').

In conclusion, these observations indicate that, in both the dorsal and ventral buds, $n k x 6.1+$ cells progressively acquire Notch signaling activity, essential for maintaining $n k x 6.1$ expression.

\section{nkx6.1 expression persists in ductal cells in the pancreas of adult zebrafish}

We then wanted to characterize $n k x 6.1$ expression in the pancreas of adult zebrafish. Immunodetection on paraffin sections through the pancreas of $\operatorname{Tg}(n k x 6.1: e G F P)$ fish that were 6 to 9 months old revealed that $n k x 6.1$ :eGFP expression persists in adult zebrafish (Fig. 7a). Comparison of endogenous Nkx6.1 protein and GFP shows that, in the adult too, $\operatorname{Tg}(n k x 6.1: e G F P)$ recapitulates the pattern of Nkx6.1 expression (data not shown). $n k x 6.1$ :eGFP expression is confined to the ducts and to isolated cells scattered throughout the exocrine tissue and was not detected in beta cells (Fig. 7a, a') or acinar cells. These $n k x 6.1: \mathrm{eGFP}+$ cells dispersed within the exocrine pancreas exhibit long cellular extensions characteristic of CACs (inset in Fig. 7a) [12]. In the adult zebrafish pancreas, as in mammals, the CACs can also be identified by Notch signaling activity [12]. Thus, to confirm the expression of $n k x 6.1$ in CACs, we used the Notch reporter line $\operatorname{Tg}(T p 1: V e n u s P e s t)$ [16], in which the destabilized Venus fluorescent protein (VenusPest) highlights cells harboring ongoing Notch activity, and which labels the CACs, as expected (Fig. 7b and inset). All Venus+ cells in the pancreas were found exclusively in the ductal system, and more particularly in the CACs and not within the ductular structures (Additional file 6: Fig. S6). Comparison of Venus with Nkx6.1 confirmed that Nkx6.1 is indeed expressed in all Venus+ CACs (Fig. 7c, c'). In contrast, while ducts also display $n k x 6.1$ expression as revealed with either $n k x 6.1$ :eGFP (Fig. 7a, a') or with the endogenous Nkx6.1 protein (Fig. 7d), they are devoid of Notch ongoing activity (white arrows pointing at Venus- ducts in Fig. 7b, d; see also Fig. 8a and Additional file 6: Fig. S6).

\section{Adult centroacinar cells display progenitor capacity in physiological conditions}

We then asked whether CACs could generate other pancreatic cell types in adult zebrafish under physiological conditions by using the double $T g(T p 1:$ VenusPest); $\mathrm{Tg}$ (Tp1:H2BmCherry) in which the stable H2BmCherry protein labels cells harboring ongoing Notch activity (Venus+ mCherry+) and cells having previously experienced Notch activity (Venus- mCherry+) [16]. This tool has been previously exploited to characterize and follow the fate of the Notch-responsive progenitors in the IPDs 

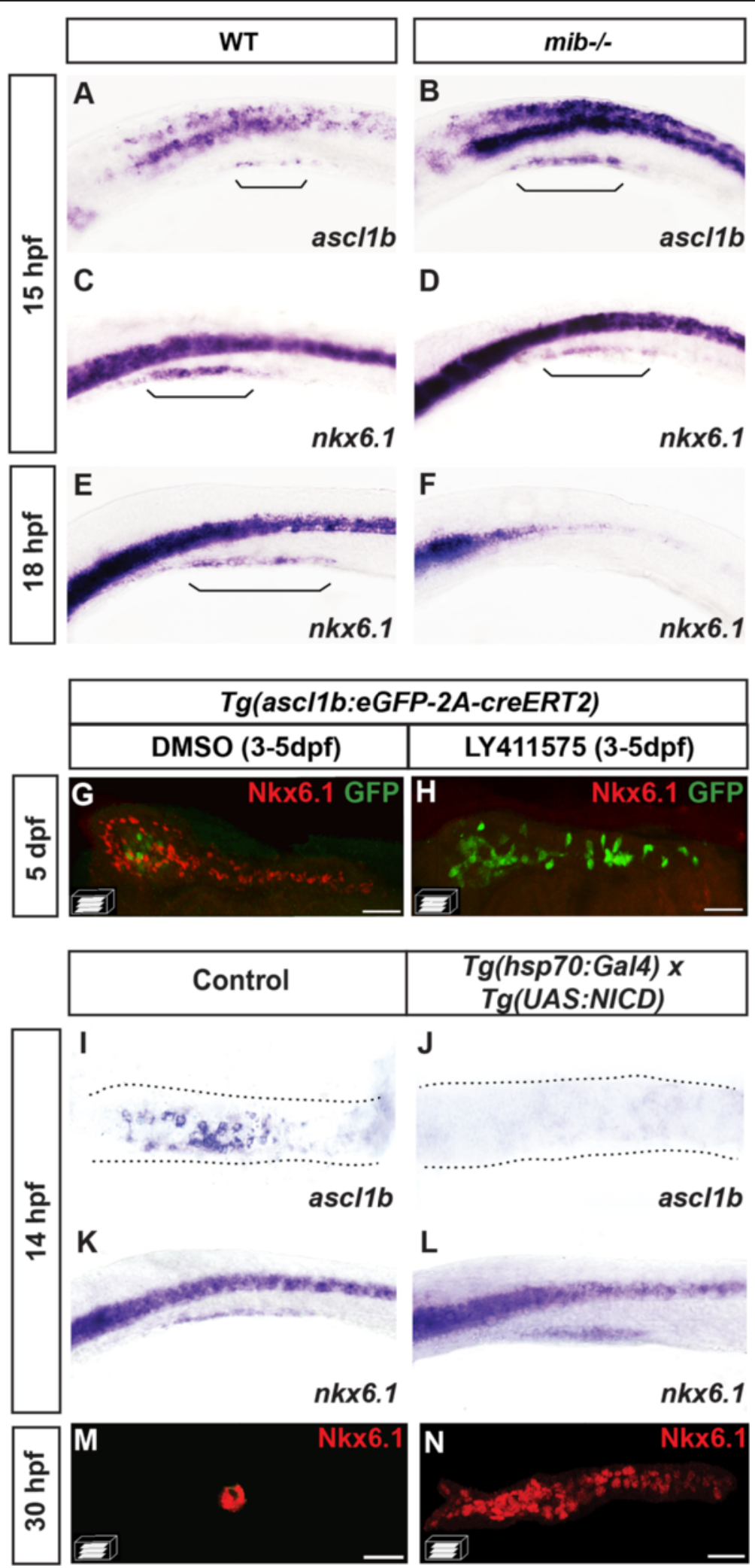

Fig. 5 (See legend on next page.) 
(See figure on previous page.)

Fig. $\mathbf{5}$ ascl1b and $n k \times 6.1$ are regulated in an opposite way by the Notch signaling pathway. a-f WISH on wild-type (WT) embryos (a, c, e) or mind bomb $\left(\mathrm{mib}^{-1-}\right)$ mutants $(\mathbf{b}, \mathbf{d}, \mathbf{f})$ with asclib $(\mathbf{a}, \mathbf{b})$ or $n k \times 6.1$ ( $\left.\mathbf{c}-\mathbf{f}\right)$ probes. Brackets delimit the pancreatic domain. $\mathbf{g}, \mathbf{h}$ Immunodetection of GFP and endogenous Nkx6.1 in 5-dpf Tg(asclib:eGFP-creER ${ }^{\text {T2}}$ ) embryos treated from 3 to 5 dpf with DMSO (g) or with the Notch signaling inhibitor, LY411575 (h). $\mathbf{i}-\mathbf{n}$ WISH with ascl1b (i, j) or $n k \times 6.1$ (k, l) probes or immunodetection of endogenous Nkx6.1 (m, $\mathbf{n})$ of double-transgenic Tg(hsp70:Ga/4) $\times$ Tg(UAS:NICD) embryos $(\mathbf{j}, \mathbf{l}, \mathbf{n})$ or of control simple-transgenic embryos $(\mathbf{i}, \mathbf{k}, \mathbf{m})$, both heat-shocked at 11 hpf for 20 min. Lateral views $(\mathbf{a}-\mathbf{f}, \mathbf{k}, \mathbf{l})$ or ventral views $(\mathbf{g}-\mathbf{j}, \mathbf{m}, \mathbf{n})$ of embryos of the indicated stages with the anterior to the left. Scale bars $=40 \mu \mathrm{m}$

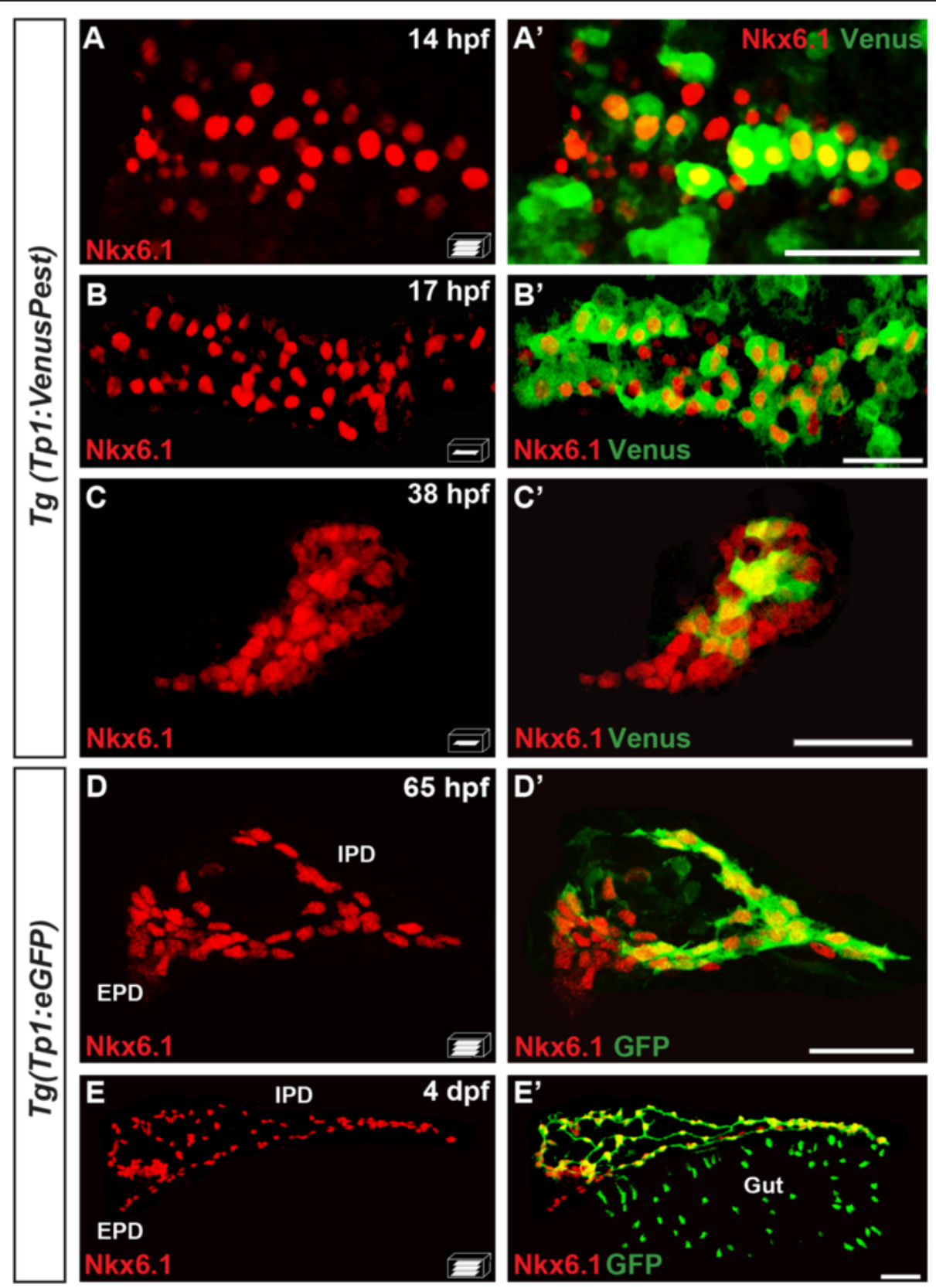

Fig. 6 The pancreatic expression domain of Nkx6.1 includes the Notch-responsive cells. Immunodetection of endogenous Nkx6.1 (red) and Venus (revealed with anti-GFP, green) in Tg(TP1:VenusPest) (a-c') or GFP in Tg(Tp1:eGFP) embryos (d-e') at the indicated stages. All views are ventral with the anterior part to the left and represent either z-plane confocal images (b-c') or confocal projection images (a, a', $\left.\mathbf{d}, \mathbf{d}^{\prime}, \mathbf{e}, \mathbf{e}^{\prime}\right)$. Scale bars $=40 \mu \mathrm{m}$. EPD extra-pancreatic duct, IPD intra-pancreatic duct 

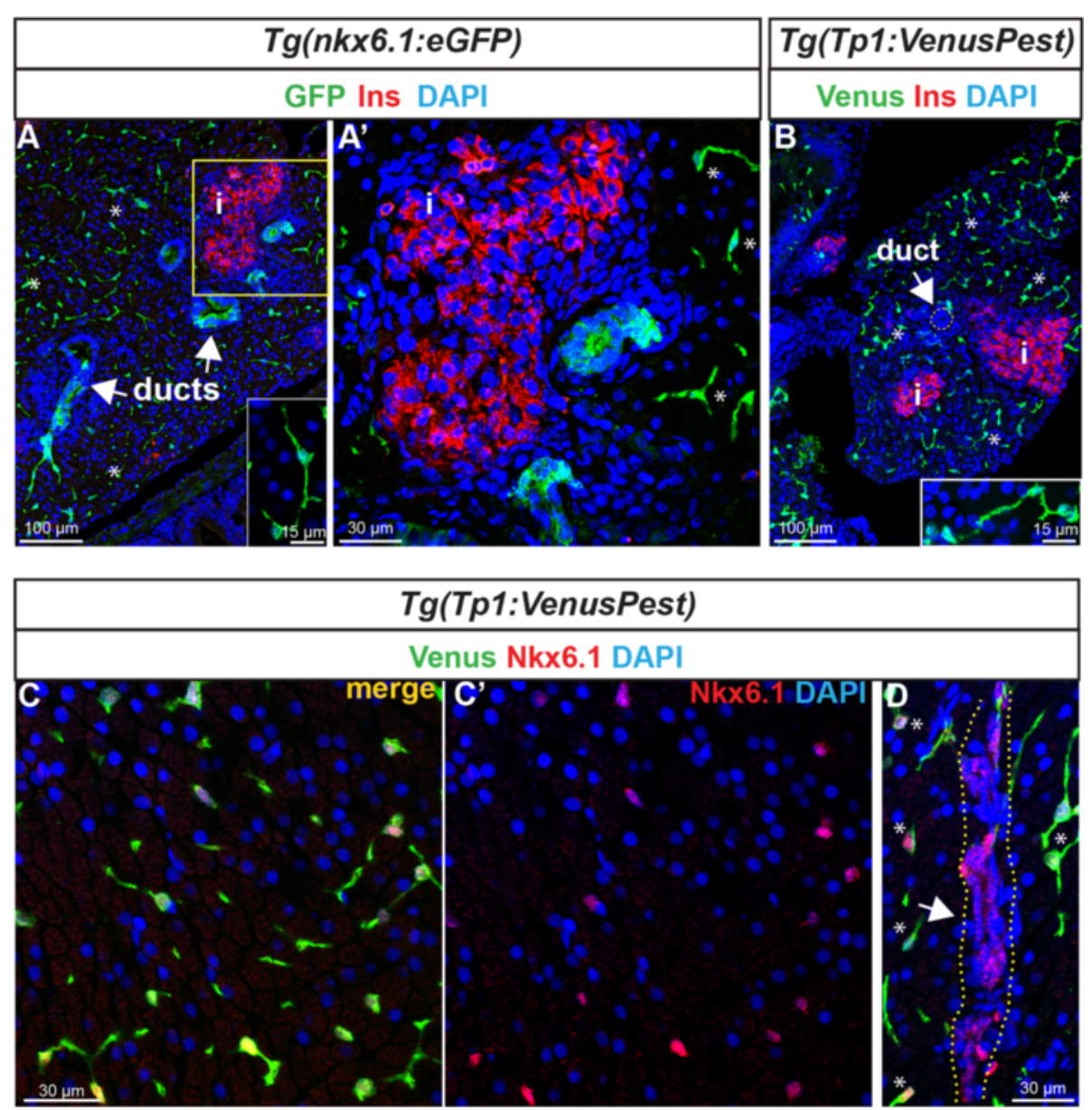

Fig. 7 Expression of $n k x 6.1$ persists in duct cells in the adult pancreas. a GFP and insulin (Ins, red) immunodetection on section through the pancreas of adult Tg(nkx6.1:eGFP) zebrafish. White arrows point to pancreatic ducts and asterisks show cells (presumably centroacinar/ terminal end duct cells (CACs) dispersed throughout the exocrine tissue). a' Close-up of the islet highlighted with Ins. b Venus (green) and Ins (red) immunodetection in Tg(Tp 1:VenusPest) showing the presence of Venus in CACs (asterisks) as previously reported [12], but not in duct cells within ductular structures (white arrow). Insets in (a) and (b) show isolated CACs. c-d Immunodetection of Venus (green) and of endogenous Nkx6.1 (red) in Tg(Tp1:VenusPest) revealing co-labeling of both markers in CACs (c, $\mathbf{c}^{\prime}$ ) while Nkx6.1 alone, but not Venus, labels the ducts. The white arrow in (d) points to a duct and asterisks indicate CACs. Dotted yellow lines delimit the duct. c' Same as (c) showing Nkx6.1 (red) and 4',6-diamidino-2-phenylindole (DAPI) only. i, islet

of larvae $[16,35]$. We thus characterized the VenusH2BmCherry+ cells in adult fish to identify the pancreatic cell types derived from CACs. In about $30 \%$ of all H2BmCherry+ cells, Notch activity was switched off (Venus-). Many of these cells, with weak H2BmCherry labeling, were identified within small ducts (Fig. 8a-c), which can be identified by the ductal marker 2F11 (Fig. 8b, c and Additional file 7: Fig. S7A-C for the separated colors and additional example) $[11,22,36]$ or by Nkx6.1 (Additional file 7: Fig. S7D), and at the tip of which reside CACs (intense $\mathrm{H} 2 \mathrm{BmCherry}$, asterisks in Fig. $8 \mathrm{c}$ and Additional file 7: Fig. S7). Furthermore, low levels of $\mathrm{H} 2 \mathrm{BmCherry}$ were also identified in some insulin-expressing cells (Fig. 8d, d'). About $4.9 \pm 2.6 \%$ $(n=4)$ of the H2BmCherry+/Notch off (Venus-) display Ins labeling. These findings show that mCherry+ terminal end duct cells and insulin-expressing cells originate from
Notch positive CACs. Overall, this reveals that a subset of the Nkx6.1+ ductal cells, the Notch-responsive CACs, can generate ductal and endocrine cells.

To determine the capacity of CACs to replicate, their proliferative status was analyzed using 5-ethynyl2 '-deoxyuridine (EdU) labeling and proliferating cell nuclear antigen (PCNA) immunodetection in $\operatorname{Tg}(T p 1:-$ VenusPest) adult fish. One day after EdU injection, a small fraction of CACs $(5.8 \pm 2.6 \%$, out of 600 counted CACs $)$ had incorporated EdU. All these EdU+ Venus+ cells also express the proliferation marker PCNA (Fig. 8e, e'). In contrast, 5 days post injection, Venus + cells that still display EdU labeling were no longer PCNA+ (Fig. 8f, f'), but still harbor characteristics of CACs, suggesting that they became post-mitotic CACs. The capacity of CACs to replicate together with their ability to undergo ductal and endocrine differentiation indicate 


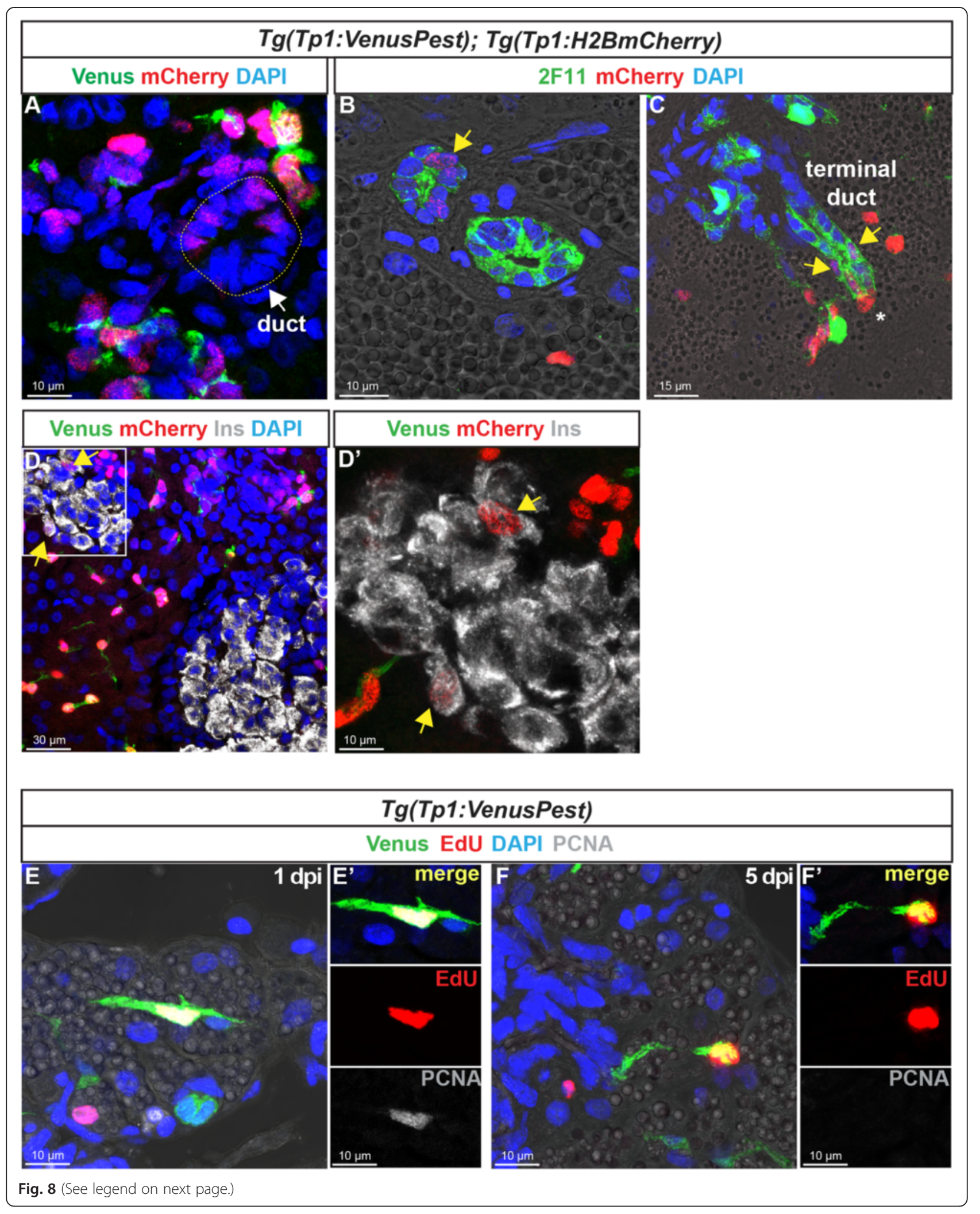


(See figure on previous page.)

Fig. 8 In the adult pancreas, Notch-responsive CACs give rise to other ductal and endocrine cells and have the capacity to replicate. Immunodetection on sections through the pancreas of adult $\operatorname{Tg}(T p 1: V e n u s P e s t) ; T g(T p 1: H 2 B m C h e r r y)$. a Comparison of Venus (green) and H2BmCherry (red) labeling showing a small duct containing $\mathrm{H} 2 \mathrm{Bm} C h e r r y+$ cells that have lost Venus. b Comparison of H2BmCherry (red) with the ductal marker 2F11 (green) showing some 2F11+ cells within a small duct co-expressing the stable H2BmCherry marker (yellow arrow). c Weak H2BmCherry labeling near the extremity of a ductular structure (terminal or intercalated duct) (yellow arrows); a CAC (intense H2BmCherry) at the tip of the terminal duct is indicated by an asterisk. $\mathbf{d}$ and $\mathbf{d}$ ' (close-up) Some H2BmCherry+ cells, devoid of Venus (Notch off) co-express the beta cell marker Ins (white) (yellow arrows). Sections acquired in the head of the pancreas, at the level of the main endocrine islet. e-f' Detection of 5-ethynyl-2'-deoxyuridine (EdU) (red), Venus (green), and proliferating cell nuclear antigen (PCNA) (white) in Tg(Tp1:VenusPest) adult fish injected with EdU. The pancreas was analyzed 20 hours (1 day post injection) (e, e') and 5 days (f, $\left.\mathbf{f}^{\prime}\right)$ after EdU injection

that they can behave as adult pancreatic progenitor/ stem cells in vivo.

\section{nkx6.1-expressing ductal cells contribute to beta cell regeneration in adult zebrafish}

The capacity of CACs to differentiate into beta cell in the normal adult zebrafish raises the question whether similar plasticity exists for regeneration. To induce beta cell regeneration, we used the $T g$ (ins:NTR-mCherry) line [1] and treatment with metronidazole (MTZ). The metronidazole is converted into a cytotoxic compound by the nitroreductase enzyme (NTR), which thereby triggers selective beta cell death by apoptosis [3]. In adult zebrafish, the ablation of beta cells causes dramatic hyperglycemia within 3 days rapidly followed by spontaneous normalization within 2 weeks and beta cell regeneration [2]. To analyze the ductal cells in the setting of beta cell regeneration, $\operatorname{Tg}(n k x 6.1: e G F P)$; $\operatorname{Tg}($ ins:NTR-mCherry) adult fish were treated with MTZ (day 1) and sacrificed at different time points during regeneration to analyze GFP and Ins on tissue sections at the level of the principal islet. The blood glucose level was measured just before sacrifice. At 3 days post treatment (dpt), ablation was total and effective as reported [2, 37]. MTZ-treated fish displayed severe hyperglycemia as expected $(>500 \mathrm{mg} /$ $\mathrm{dl}, n=10$ fish) while the glycemia of non-treated fish was at normal values of $61 \pm 19 \mathrm{mg} / \mathrm{dl}(n=11)$. Immunolabeling at $3 \mathrm{dpt}$ indicated total beta cell ablation as manifested by the absence of insulin staining in the pancreas of MTZ-treated $\operatorname{Tg}(n k x 6.1: e G F P) ; T g(i n s: N T R-m C h e r r y)$ fish (Fig. 9a, b). At 9 dpt, the first new beta cells have started to reappear (Fig. 9c). Glycemia was still above normal values but nonetheless had decreased $(145 \pm 31 \mathrm{mg} / \mathrm{dl}$, $n=2)$. The principal islet in the treated fish still showed very weak insulin expression but a few new beta cells (about $5 \%$ of the islet cells, compared to $40-70 \%$ in control fish) can be detected in the principal islet as well as throughout the exocrine tissue as isolated cells or as small clusters of Ins-expressing cells next to CACs and ductal cells marked by GFP (Fig. 9c, yellow arrows). The presence of insulin-expressing cells at $9 \mathrm{dpt}$ is indicative of beta cell regeneration. Interestingly, some of the regenerating insulin+ cells displayed weak GFP staining $(7.9 \pm 3.2 \%$ of the Ins+ cells, 452 counted cells, $n=2$ fish) (Fig. 9f). These cells were found next to strongly GFP-labeled ductal nkx6.1:eGFP cells. GFP+ Ins + cells were also detected at $21 \mathrm{dpt}(11.1 \pm 4.3 \%$ of the Ins+ cells, 940 counted cells, $n=2$ fish) (Fig. 9g). At this stage, a large number of beta cells have recovered (about $30-50 \%$ of islet cells, Fig. 9d) [2, 37] and glycemia was normalized ( $57 \pm 9 \mathrm{mg} / \mathrm{dl})$. In contrast, insulin+ cells never harbor GFP labeling in non-treated fish (Fig. 9e). Thus, by using the same approach of short-term tracing of nkx6.1:eGFP+ cells as in embryos, this finding strongly suggests that ductal nkx6.1:eGFP+ cells contribute to regenerated beta cells.

Next we assessed proliferation in $\operatorname{Tg}(n k x 6.1: e G F P)$; Tg(ins:NTR-mCherry) in response to beta cell ablation. Three days post treatment, $n k x 6.1$ :eGFP ductal cells showed increased proliferation as illustrated with PCNA (Fig. 9h, i). This was observed not only within ductal structures as previously described ([2] and not shown) but also for CACs. These observations suggest that ductal cells with pancreatic progenitor properties activate proliferation prior to differentiation into beta cells during regeneration.

\section{Transcriptomic analysis of adult $n k x 6.1+$ pancreatic ductal cells}

To get a comprehensive characterization of pancreatic $n k x 6.1+$ ductal cells in adult zebrafish, we determined their transcriptome landscape. Ductal GFP+ cells were isolated from dissected pancreases of adult $\operatorname{Tg}(n k x 6.1: e G F P)$ fish (with $\sim 95 \%$ purity) and used in RNA-seq experiments. The expression of 15,888 genes could be detected in the ductal transcriptome (complete data available at [38]), in which many genes already known to be expressed in pancreatic ducts in either zebrafish or mammals were found at high expression level, such as sox $9 b$, hnf1ba, onecut1/hnf6, cftr, cdh17, ca2, and ctgfa in addition to $n k x 6.1$ (Fig. 10). We also detected expression of fgfr4 and $s d c 4$, recently proposed as novel ductal markers in the murine embryonic pancreas [39]. In contrast to these ductal genes, the acinar markers ptfla and rbpjl, the pan-endocrine markers pax6b and isl1, and the lineage specific genes $m n x 1$ and arx were either not detected or detected at extremely low levels in the $n k x 6.1: \mathrm{eGFP}+$ ductal cells transcriptome (Fig. 10), 


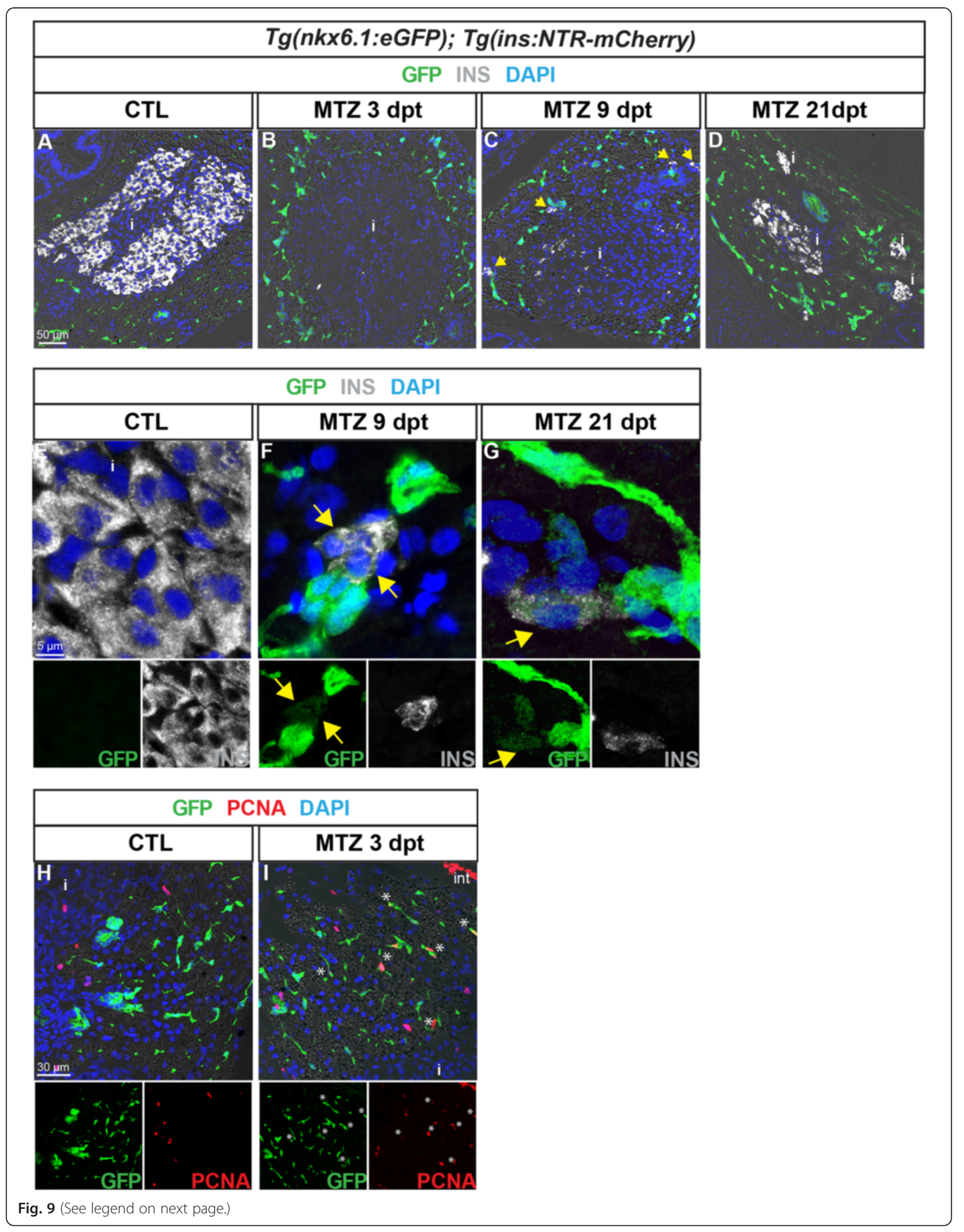


(See figure on previous page.)

Fig. 9 nkx6.1:eGFP+ cells proliferate and differentiate into new insulin-expressing cells after beta cell specific ablation. GFP (green) and Ins (white) labeling in the pancreas of Tg(nkx6.1:eGFP); Tg(ins:NTR-mCherry) adult fish. a, b Non-treated fish (CTL, a) show intense Ins staining in beta cells, while Ins+ cells are not detected $3 \mathrm{dpt}$ with MTZ, indicating efficient ablation (b). Note that debris of one beta cell (Ins+) is observed in the islet. c At 9 dpt, Ins-expressing cells start to be detected in the principal islet and in extra-insular locations close to ductal GFP+ cells (yellow arrows). d At 21 dpt, islets show intense Ins staining consistent with beta cell recovery. $\mathbf{e}-\mathbf{g}$ (and separate channels) While GFP is never detected in beta cells of control fish (e), some regenerating Ins+ cells display weak GFP labeling at both stages of regeneration analyzed, i.e. at 9 dpt (f) and 21 $\mathrm{dpt}(\mathbf{g})$. h, i (and separate channels) Beta cell ablation triggers proliferation of CACs as shown at $3 \mathrm{dpt}$ (h) compared to CTL (i) (asterisks). i islet

underscoring the accuracy of our fluorescence-activated cell sorting (FACS) cell preparations. In contrast to mouse or human adults in which the embryonic pancreatic progenitor marker $P d x 1$ is not expressed in the pancreatic ducts in normal condition, $n k x 6.1: \mathrm{eGFP}+$ duct cells of healthy zebrafish display a robust expression of $p d x 1$. Comparison of the duct transcriptome with those of pancreatic acinar and endocrine cells (manuscript in preparation) highlighted 3,684 genes with preferential expression in duct cells. Among them, 293 duct-specific genes were identified with strong enrichment ( $\geq 16$-fold) and low expression in the other pancreatic cells (Additional file 8: Table S1), in which we find sox $9 b$, onecut1/hnf6, cdh17, ctgfa, and $n k x 6.1$, corroborating their status as duct-specific markers. Various components of the Notch signaling pathway could also be identified, namely notch2, and different Hairy and enhancer of split-related genes (her6, her9, and her15.1), confirming that a subpopulation of $n k x 6.1+$ cells (the CACs) experiences Notch activity. In addition to genes involved in the Notch signaling cascade, our analyses also identified novel duct-specific markers such as $i d 2 a$, encoding for a HLH transcription factor, which we also detected coexpressed with $s o x 9 b$ by WISH in 3-dpf larvae (Additional file 9: Fig. S8), and several components of the Wnt pathways such as the Wnt and SFRP ligands sfrp5, sfrp3/frzb, and $w n t 7 b b$ (Additional file 8: Table S1). At a lower expression level, we could also detect the Wnt receptor $f z d 7 a$, which was the only Wnt receptor significantly expressed in the adult pancreas $(565 \pm 106$ normalized counts with 68 -fold enrichment in the ducts). These observations suggest that Wnt signaling may play an important role in adult ducts.

Strikingly, the only endocrine transcription factor that displayed substantial expression (>1000 counts) and enrichment in the ductal transcriptome was the proendocrine gene ascl1b (Fig. 10). As ascl1b specifically marks the endocrine precursors during pancreas development, these data support our observation that, within

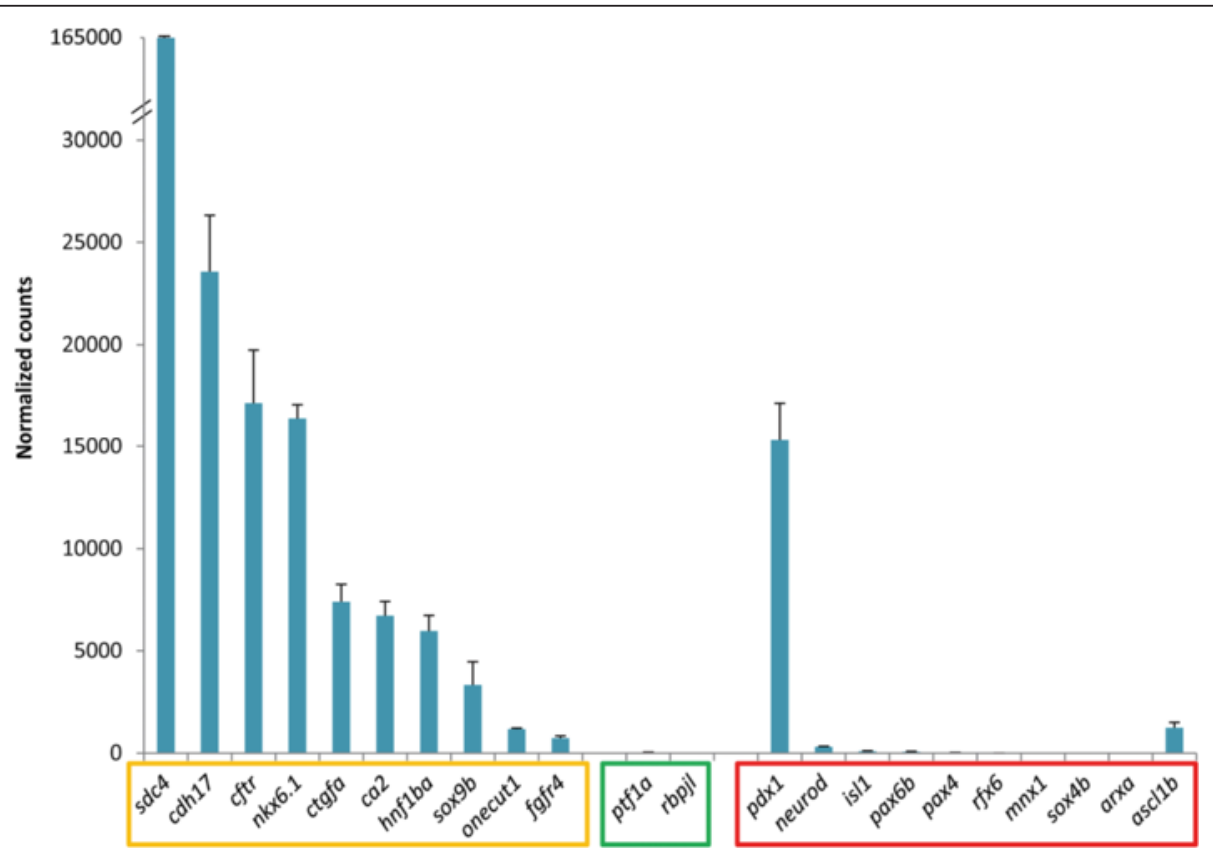

Fig. 10 Expression of known pancreatic markers in the transcriptome of adult nkx6.1:eGFP cells. Expression values are expressed as normalized counts. High expression of known ductal genes (orange box) is detected while acinar (green box) and endocrine (red box) markers display low abundance, with the exception of $p d x 1$ and asclib (see text) 
the $n k x 6.1: \mathrm{eGFP}+$ cell domain, some cells activate a pro-endocrine differentiation program in normal adult zebrafish.

\section{Discussion}

In this study, we determined during development the fate of two pancreatic cell populations marked by $n k x 6.1$ and $a s c l 1 b$ and found that $n k x 6.1+$ cells are bona fide multipotent pancreatic progenitors while ascl1b+ cells represent committed endocrine precursors. We found also that $n k x 6.1$ is maintained in adult zebrafish in ducts and $\mathrm{CAC} /$ terminal end duct cells, which we show still have the potential to give rise to endocrine cells in normal (non-diabetic) animals. The progenitor potency of adult $n k x 6.1+$ cells is also reflected in their transcriptome through the expression of several pancreatic progenitor markers and by their capacity to generate new beta cells after beta cell ablation.

Although ascl1b marks the endocrine precursor cells, as opposed to $n k x 6.1$, which is the first multipotent pancreatic progenitor marker known to date, it is surprising to note that this transcription factor begins to be expressed in the prospective pancreatic region at $10 \mathrm{hpf}$ [15], i.e. more than 1 hour before the appearance of $n k x 6.1$ (11.5 hpf) [18]. $P d x 1$, known in the mouse to be also expressed in the multipotent pancreatic progenitors, appears even later (14 hpf [6]). This brings the interesting concept that the first cells in the pancreatic anlagen acquire an endocrine identity before acquiring a pancreatic identity, suggesting that the mechanisms controlling pancreatic and endocrine identity are not necessary linked and can act in parallel. This situation seems to be restricted to the dorsal bud as, later, $n k x 6.1$ is first expressed during the formation of the ventral bud and $a s c l 1 b$ is then detected in endocrine committed cells. This peculiar situation could be related to the different lineage potential of the dorsal versus ventral bud cells. Indeed the dorsal bud gives rise exclusively to endocrine cells while the ventral bud is able to give rise to all pancreatic cell types $[4,40]$.

After a transient overlapping expression in the dorsal pancreatic anlagen, $n k x 6.1$ - and ascl1b-expressing domains segregate progressively. Cross-repressive interactions between lineage-determining transcription factors have been proposed as a molecular mechanism for establishing lineage allocation in several tissues [41-43]. We show that such a mechanism does not occur here, as we could not observe any crossrepression between $n k x 6.1$ and ascl1b in the dorsal bud when analyzing both mutants. The segregation can instead be explained by the opposite effects of the Notch signaling pathway on $n k x 6.1$ and $a s c l 1 b$ expression. By loss- and gain-of-function experiments, we definitely prove that $a s c l 1 b$ is repressed, while $n k x 6.1$ is maintained, by Notch signaling in both the dorsal and ventral buds. The importance of Notch signaling in regulating $n k x 6.1$ expression has been also shown in mice where disruption of Notch signaling results in the loss of the pro-trunk determinant Nkx6.1 and the acquisition of pro-acinar identity [44]. Direct binding of RBPJ-K to the $N k x 6.1$ promoter supports a direct role of Notch signaling in the expression of $N k x 6.1$. In contrast, the initiation of $n k x 6.1$ expression is independent of Notch signaling in both zebrafish pancreatic buds. This is in accordance with our findings that Nkx6.1+ cells progressively become Notch-responsive PNCs being found only in a subpopulation of the Nkx6.1+ cells at the beginning of the formation of both the dorsal and ventral buds. This could explain why the PNCs, found in a subdomain of the ventral bud at an early stage, can differentiate only into ductal and endocrine cells, while $n k x 6.1$-expressing cells give rise to all pancreatic cell types. As $n k x 6.1$ and $p t f 1 a$ are initially co-expressed in the ventral bud primordium ([20] and our unpublished data), we can hypothesize that the $n k x 6.1+/ p t f 1 a+/$ Notch on cells give rise to the ductal and late endocrine cells while the $n k x 6.1+/ p t f 1 a+/$ Notch off cells will give rise to the acinar cells. This model appears to contradict the data of Wang et al. [7] showing that the ventral bud primordium consists of two nonoverlapping cell populations: a $p t f 1$-expressing domain and a Notch-responsive progenitor core. It is possible that this discrepancy is due to the tools used to label the Notch-responsive cells: in our study, we used the Tp1bglob:VenusPest transgenic line allowing the detection of cells with current Notch activity, while Wang and collaborators used the Tp1bglob:hgmb1-mCherry line, which could show a delay in mCherry detection.

In adult zebrafish, $n k x 6.1$ expression persists in the pancreas where it is specifically restricted to the ducts. This situation is different in the mouse as $N k x 6.1$ persists in beta cells but not in adult ducts. In zebrafish, it is $n k x 6.2$, an $n k x 6.1$ homolog with functional equivalence $[18,45]$, which is expressed specifically in beta cells [18], suggesting that the Nkx6 function in beta cells is fulfilled by Nkx6.2 in zebrafish. Persistence of $n k x 6.1$ expression in the zebrafish ducts is also associated with persistence of another pancreatic progenitor marker, $p d x 1$, whose expression is also restricted to beta cells in mammals. Combined with other hallmarks of embryonic pancreatic progenitors such as sox $9 b$, hnf1ba, and Notch signaling components, the expression of genes $n k x 6.1$ and $p d x 1$ suggests that at least some ductal cells behave as pancreatic progenitors in adult zebrafish. Moreover, detection of the endocrine precursor marker ascl1b in the adult $n k x 6.1: e G F P+$ ductal cell transcriptome is consistent with some ductal cells initiating an endocrine differentiation program and transiently expressing ascl1b, 
even in physiological conditions. The progenitor potential of ductal cells is fully revealed in the setting of beta cell regeneration where $n k x 6.1: e \mathrm{GFP}+$ cells show increased proliferation as well as the ability to differentiate into new beta cells. The adult pancreatic progenitors contributing to beta cell regeneration could be the CACs, as supported by our observation that they are able to replicate and to generate other ductal cells as well as endocrine beta cells. On the other hand, we cannot exclude the possibility that other ductal cells also contribute to regeneration based on $\operatorname{Tg}(n k x 6.1$ :eGFP), which labels more broadly the ducts. Nevertheless, although the tool we used here to monitor lineage tracing presents some limitations (based on the persistence of GFP in assessing the short-term lineage tracing of $n k x 6.1$-expressing cells), during the revision of our manuscript, a study by Delaspre et al. [37] established through CRE-based genetic labeling that Notch-responsive cells give rise to regenerated beta cells in adult zebrafish. Our data are in full accordance with their findings, and support the conclusion that ductal cells, possibly CACs, possess regenerative capacity. To determine whether CACs only or other ductal cells contribute to beta cell regeneration will require other genetic lineage tracings with markers expressed only in ductular structures and not in CACs.

In contrast to mammals, the adult zebrafish has the remarkable capacity to regenerate its beta cells rapidly and spontaneously following their selective destruction [2]. Our findings showing that ductal cells, such as CACs, behave as pancreatic progenitors/stem cells in normal (non-diabetic) adult animals and during regeneration strongly suggest that they could also constitute a source of regenerated beta cells in diabetic mammalian models. In the mouse, adult murine CACs display endocrine and exocrine progenitor potential in vitro with self-renewing ability [46], but evidences of their potential in vivo as progenitors of endocrine cells are missing. Indeed, CRE-based cell tracing of Hes1+ terminal duct cells/CACs in adult mice failed to show any islet progenitor capacity while these cells seemed to contribute to the ductal tree [47]. One explanation for this difference would be that while zebrafish CACs present pancreatic progenitor activity, mammalian CACs have retained a very limited capacity in vivo, which could not be evidenced in the mouse model of beta cell regeneration used. Another explanation would be that CACs form a heterogeneous cell population. This latter hypothesis could be verified by the analysis of the expression of different ductal markers identified in our transcriptome, which could help determine the existence of different ductal cell subpopulations.

A perspective of our work would be to examine thoroughly in zebrafish ductal cells in physiological conditions and during beta regeneration to identify mechanisms that could then be harnessed to promote beta cell regeneration in mammals. A first clue is provided by the fact that $N k x 6.1$ and $P d x 1$ are normally not expressed in mammalian duct cells, in contrast to zebrafish. It is therefore tempting to hypothesize that inducing the expression of these two factors in the pancreatic ducts in the adult mouse or human could enhance their progenitor potential. Consistent with this hypothesis, driving ectopic $P d x 1$ expression is already harnessed for transdifferentiating different cells into beta cells in vivo (liver cells, pancreatic acinar cells, ...) [48-51] and for transdifferentiating pancreatic duct cells into beta cells in vitro [52]. Interestingly, the ability of $P d x 1$ to reprogram liver cells into beta cells is substantially increased by the combined action of Nkx6.1 [53], which potentiates induction of the early pancreatic master genes Neurog3 and Isl1.

In the zebrafish larvae, two sources have been described for the regeneration of beta cells: progenitor cells in the developing ducts [35] and alpha cells [54]. From our data in adults, we cannot rule out the contribution by other cell types than ductal cells. Indeed, in addition to increased proliferation of ductal cells, some islet cells show also increased proliferation after beta cell destruction ([2] and our data not shown) suggesting the involvement of other pancreatic, and possibly endocrine, cell types in regeneration. These possibilities remain to be validated in the adult zebrafish.

Our transcriptomic characterization of $n k x 6.1+$ cells in normal non-regenerating adult zebrafish identified many of the ductal markers known throughout species, showing that many of these genes and their expression are conserved between zebrafish and mammals, as for example, different markers of Notch signaling [55-57], and $s d c 4$ and $f g f r 4$. These latter two genes have been previously proposed as ductal markers in the mouse embryo [39]. Additional markers were also identified here, notably $i d 2 a$. Id 2 has been shown to be expressed in ductal epithelial cells of IFNYNOD mice, a model of a regenerating pancreas harboring hyperplasic ducts, and to be involved in their expansion [58]. We demonstrate here its expression in ducts in larvae and in healthy adult pancreas, which may correlate with a role in development and in ductal constitutive homeostasis. Our findings also reveal that, besides the Notch pathway, ductal cells specifically express various components of Wnt signaling pathways. The expression of the $f z d 7 a$ receptor, of two secreted Wnt antagonists, sfrp 5 and frzb/sfrp3, and of the agonist wnt $7 b b$ suggests a complex control of the activity of the Wnt pathway(s) in adult pancreatic ducts. Whether and how these different factors orchestrate pancreatic duct development, homeostasis, and function remains to be determined. Numerous crosstalks between the Notch and the Wnt/beta-catenin 
pathways occur during development, tissue homeostasis, and disease, notably by regulating the balance of stem cells and differentiated cells (reviewed in [59]). During pancreas development, beta-catenin controls the patterning of multipotent versus bipotent embryonic pancreatic progenitors in the mouse, in part, by inhibiting Notch signaling [60]. Our findings raise the question whether similar interactions shape the fate decision of progenitors/stem cells in the adult pancreas.

\section{Conclusions}

We have developed transgenic tools enabling the characterization of $n k x 6.1+$ and ascl1b+ progenitor cell populations and showed that, in the zebrafish embryo, $n k x 6.1+$ cells are multipotent pancreatic progenitors, while $a s c l 1 b+$ cells represent committed endocrine precursors. In adult zebrafish, $n k x 6.1$ expression persists exclusively in the ductal tree, notably in CACs. Transcriptomic profiling of adult $n k x 6.1+$ ductal cells reveals hallmarks of embryonic pancreatic progenitors and identifies novel ductal markers. Our data also strongly suggest that adult zebrafish ductal cells, possibly CACs, possess regenerative capacity. Further characterization of ductal cells in this animal model should bring new insight into regeneration in mammals and open up new perspectives for anti-diabetic therapies.

\section{Methods}

\section{Zebrafish maintenance, mutant and transgenic lines, and LY411575 treatment}

Zebrafish (Danio rerio) were raised and cared for according to standard protocols [61]. All animal work has been conducted according to national guidelines and all animal experiments described herein were approved by the ethical committee of the University of Liège (protocol numbers 371, 1285, and 1662). Wild-type embryos from the $A B$ strain were used and staged according to Kimmel [62]. Homozygous mind bomb mutants were obtained by mating heterozygous fish for the $\left(\mathrm{mib}^{\text {ta52b }}\right.$ ) allele [63]. The following transgenic lines were used: $\operatorname{Tg}$ (hsp70l:Gal4)1.5kca4 abbreviated $\operatorname{Tg}$ (hsp:Gal4) and $\operatorname{Tg}(\text { UAS:myc-Notch1a-intra })^{k c a 3}$ abbreviated $\operatorname{Tg}(U A S: N I C D)$ [33], $\operatorname{Tg}(T p 1 \text { bglob:eGFP })^{\text {um14 }}$ abbreviated $\operatorname{Tg}(T p 1: e G F P)$ [12], Tg(TP1bglob:VenusPest $)^{S 940}$ abbreviated $\operatorname{Tg}($ TP1:VenusPest $) \quad[16], \quad T g$ (Tp1bglob:H2BmCherry) ${ }^{5939}$ abbreviated $\operatorname{Tg}(\mathrm{Tp} 1: H 2 B m$ Cherry) [16], $\operatorname{Tg}($ ubi:loxP-EGFP-loxP-mCherry) abbreviated ubi:Switch [28], Tg(ubi:loxP-AmCyan-loxP-ZsYellow) abbreviated ubi:CSY [27], Tg(ins:NTR-mCherry) [1], and $\operatorname{Tg}(n k x 6.1: e G F P) ; \operatorname{Tg}($ ins:NTR-mCherry).

The LY411575 treatment was performed by incubating the embryos during the indicated period with a $10-\mu \mathrm{m}$ LY411575 solution (Medchemexpress), replaced every day.

\section{Generation of BAC transgenic lines}

The polymerase chain reaction ( $\mathrm{PCR}$ ) primers used to generate the constructs are listed in Additional file 10: Table S2. The BAC:nkx6.1 (Imagenes, DKEY-173 K2) DNA was introduced by electroporation into SW102 E. coli (derived from DY380) [64]. These bacteria contain the lambda prophage recombineering system and a galactose operon where the galactokinase gene (galK) has been deleted. The eGFP gene was inserted into exon 1 of $n k x 6.1$, replacing the beginning of the $n k x 6.1$ open reading frame (amino acids (aa) 1 to 149) using a twostep positive and negative galk selection [25, 64, 65]. During the first step, the cassette containing the galk gene was amplified by PCR with the pair of primers $\mathrm{O} 180 \mathrm{~F}$ and $\mathrm{O} 253 \mathrm{R}$, containing at the $5^{\prime}$ end 50 bases identical to the $n k x 6.1$ sequence to allow homologous recombination and electroporated into the bacteria SW102 containing BAC:nkx6.1. Only recombinant bacteria are able to grow on minimal medium containing galactose as carbon source. During the second step, the galK gene was replaced by the eGFP gene. The eGFP cassette was amplified by PCR with the primers O186F and O256R containing at the $5^{\prime}$ end the same 50 bases identical to the $n k x 6.1$ sequence to allow homologous recombination and the eGFP sequences to anneal to the eGFP cassette. After electroporation, the bacteria were plated on minimal medium containing two-deoxy-D-galactose (DOG), a galactose analogue that after phosphorylation by GalK, becomes toxic. Only bacteria that have lost the galk gene survived on DOG-containing medium. To facilitate the insertion of the BAC in the genome of zebrafish, the $i$ Tol2 cassette was also inserted into the backbone of BAC:nkx6.1-eGFP [24, 25, 65]. The iTol2 cassette was amplified by PCR with the pair of primers $\mathrm{O} 215 \mathrm{~F}$ and $\mathrm{O} 216 \mathrm{R}$. The final construct (BAC:nkx6.1-eGFP) was purified with Nucleobond $^{\circ}$ BAC100 (Macherey-Nagel) and injected into the cytoplasm of one-cell-stage zebrafish embryos together with the mRNA for the transposase. The embryos and larvae were screened for GFP expression and the fluorescent injected fish were raised to adulthood and the offspring were screened for fluorescence. The transgenic line obtained was abbreviated to $\operatorname{Tg}(n k x 6.1: e G F P)$ in the article.

To generate the (ascl1b:eGFP-2A-creER ${ }^{T 2}$ ) transgenic line, we used BAC:ascl1b (Imagenes, DKEY-265 N18) spanning from $61 \mathrm{~kb}$ upstream and $89 \mathrm{~kb}$ downstream of the ascl1b gene. The GFP-2A-creER ${ }^{T 2}$ cassette was inserted into exon 1 of ascl1b, replacing the beginning of the $a s c l 1 b$ open reading frame (aa 1 to 163) using the same two-step positive and negative galk selection as described above. For the first step, the cassette containing the galk gene was obtained by PCR using the primers $\mathrm{O} 275 \mathrm{~F}$ and $\mathrm{O} 276 \mathrm{R}$ and for the second step, the 
GFP-2A-creER ${ }^{T 2}$ cassette was amplified using the primers $\mathrm{O} 277 \mathrm{~F}$ and $\mathrm{O} 278 \mathrm{R}$.

\section{OHT treatment for creER ${ }^{\mathrm{T} 2}$ induction}

4-Hydroxytamoxifen (4OHT, Sigma H7904) was dissolved in DMSO as a stock solution of $10 \mathrm{mM}$ and kept in single-use aliquots in the dark at $-70{ }^{\circ} \mathrm{C}$. A working concentration of $10 \mu \mathrm{M} 4 \mathrm{OHT}$ was demonstrated to lead optimally to Cre-mediated recombination without causing deleterious development defects. Embryos were treated five times from 11 to $15 \mathrm{hpf}$ in E3 containing 10 $\mu \mathrm{M} 4 \mathrm{OHT}$ and kept in the dark at $28{ }^{\circ} \mathrm{C}$. After the treatments, the embryos were washed in fresh E3 and fixed at $48 \mathrm{hpf}$ or $72 \mathrm{hpf}$.

\section{CRISPR/cas9 genome mutagenesis}

The $n k x 6.1$ and ascl1b mutant lines were generated by CRISPR/Cas9 technology essentially as described previously $[31,66]$. The targeted sites were selected using the ZiFiT software package [67] in the first exon of $n k x 6.1$ (CCAAACCCCTGACAGAGCTTC) before the homeodomain coding region and in the first exon of ascl1b (GGAGACGCTGCGCTCCGCCGTGG) corresponding to the helix-loop-helix coding domain. The selected oligonucleotides were inserted into the plasmid DR274 (Addgene) and the gRNA (guide RNA) was synthesized by in vitro transcription using T7 RNA polymerase. Fertilized zebrafish eggs were injected with about $1 \mathrm{nl}$ of a solution containing $50 \mathrm{ng}$ of gRNA and $300 \mathrm{ng}$ of nlszCas9-nls mRNA obtained by transcription of the plasmid pT3TS-nCas9n (Addgene). The efficiency of mutagenesis was verified by genotyping using Heteroduplex Migration Assays [68] after amplification of targeted genomic sequences. Injected embryos were raised until adulthood and crossed with wild-type fish to generate heterozygote mutant F1 fish. Fish harboring frameshift mutations were kept and used to raise F2 mutant lines, i.e. the $a s c l 1 b^{u l g-M 2 C}$ and $n k x 6.1^{u l g-M 5}$ lines carrying, respectively, 11 and seven nucleotide deletions.

\section{Whole mount in situ hybridization, whole mount immunohistochemistry, and immunohistochemistry on paraffin sections}

Double fluorescent and visible WISH were performed as previously described $[69,70]$ with the following probes: ascl1b [15, 71], nkx6.1 [18, 72], somatostatin 2 (sst2) [73], and try [6].

Immunohistochemistry (IHC) on whole-mount embryos was performed as described [15]. For IHC on paraffin sections with adult tissues, adult fish between 6 and 9 months old were fixed in $4 \%$ PFA (paraformaldehyde) overnight at $4^{\circ}$ after euthanasia and opening of the abdominal skin. The digestive tract was then dissected and embedded in paraffin following standard procedures. Then, $5-\mu \mathrm{m}$ sections were collected through the head of the pancreas at the level of the main pancreatic islet. Immunodetection was performed after standard antigen retrieval.

The antibodies used were: polyclonal rabbit antimCherry/dsRed (Living Colors DsRed Polyclonal Antibody, Clontech) 1:500, polyclonal rabbit anti-ZsYellow (Living Colors anti-RCFP polyclonal pan from Clontech) 1:300, chicken anti-GFP (Aves lab) 1:1000, mouse monoclonal anti-Nkx6.1 (clone F55A10) 1:20, mouse monoclonal anti-Isl1 (Hybridoma bank) 1:50, guinea pig anti-insulin (Dako) 1:500, mouse anti-glucagon (Sigma) 1:300, polyclonal rabbit anti-somatostatin (MP Biomedicals) 1:300, mouse anti-PCNA (Sigma) 1:1000, mouse 2F11 mAb (Abcam) 1:1000, and Alexa Fluor secondary antibodies (Invitrogen). Venus was detected with anti-GFP. Finally, 4',6-diamidino-2-phenylindole (DAPI) was used as nuclear staining.

Images were acquired with a Leica SP2 or SP5 confocal microscope and processed with Imaris 7.2.3 and Photoshop CS5. To count the Tp1:H2BmCherry+ cells expressing insulin, the total number of pancreatic mCherry+/Venus- and mCherry+/Ins+ cells was calculated for six sections every $15 \mu \mathrm{m}$ for four fish.

\section{EdU injection and detection in adult zebrafish}

A 12.5-mM EdU solution in DPBS (Dulbecco's Phosphate Buffer Saline) containing 0.25 \% DMSO was injected intraperitoneally in 6-9-month-old fish at $100 \mu \mathrm{g} / \mathrm{g}$ body weight after anesthesia in tricaine methane sulfonate. Fish were then sacrificed and fixed in $4 \%$ PFA. EdU (Click-iT ${ }^{\circ}$ Labeling Technologies, Life Technologies) incorporation was detected on paraffin sections with Alexa Fluor555 before proceeding to IHC detection.

To count EdU+ cells, the total number of pancreatic Venus+ CAC and Venus+/EdU+ cells was calculated in three to six sections every $15 \mu \mathrm{m}$ for four fish.

\section{Induction of beta cell ablation in adult zebrafish}

Adult $\operatorname{Tg}(n k x 6.1: e G F P) ; \operatorname{Tg}($ ins:NTR-mCherry) zebrafish between 6 and 9 months old were treated in fish water containing $10 \mathrm{mM} \mathrm{MTZ} \mathrm{(three} \mathrm{to} \mathrm{four} \mathrm{fish} \mathrm{per} 500 \mathrm{ml}$, Sigma 3761) for 20 hours at $28{ }^{\circ} \mathrm{C}$. Then the water was replaced twice before re-integration into the system.

Fish were anesthetized with tricaine and their glycemia was measured using the Accu-Chek Aviva glucometer system (Roche Diagnostics) with blood collected at the level of the tail. To minimize variations, the fish were fasted for 24 hours before measurement. After decapitation, the whole fish were fixed in $4 \%$ PFA overnight at $4{ }^{\circ} \mathrm{C}$. The digestive tract was then dissected prior to paraffin embedding.

\section{FACS purification of ductal cells}

The pancreas of three to five $\operatorname{Tg}(n k x 6.1: e G F P)$ adult fish (6-9 months old) were dissected and collected 
in HBSS with calcium. Dissociation was performed in HBSS (Hank's Balanced Salt Solution) with $\mathrm{Ca}^{2+} / \mathrm{Mg}^{2+}$ supplemented with $1 \mathrm{mg} / \mathrm{ml}$ collagenase IV (Life Technologies 17104-019) and collagenase P (Roche 1121386501) and 1.5 $\mathrm{mg} / \mathrm{ml}$ dispase II (Life Technologies 17105-041) for $20 \mathrm{~min}$ at $30{ }^{\circ} \mathrm{C}$. After several washes in HBSS without $\mathrm{Ca}^{2+} / \mathrm{Mg}^{2+}$, a single-cell suspension was obtained by Tryple Select $1 \times$ incubation for $10 \mathrm{~min}$. Dissociation was stopped by HBSS without $\mathrm{Ca}^{2+} / \mathrm{Mg}^{2+}$ containing $1 \%$ BSA (bovine serum albumin) and 2 mM EDTA (ethylenediaminetetraacetic acid). GFP+ cells were isolated on FACS Aria II under purity mode and the purity of the sorted cells was confirmed for a small fraction with an epifluorescence microscope ( 95\%). Cells were immediately lyzed in $3.5 \mu \mathrm{l}$ of reaction buffer (SMARTer Ultra Low RNA kit for Illumina sequencing, Clontech) and stored at $-80{ }^{\circ} \mathrm{C}$. Three independent replicates were generated from 6-9-month-old fish.

\section{cDNA synthesis and library preparation}

cDNA synthesis was performed using the SMARTer Ultra Low RNA kit for Illumina sequencing (Clontech) according to the manufacturer's recommendations. Then 3000-5000 sorted GFP+ cells were directly lyzed in 3.5 $\mu \mathrm{l}$ of reaction buffer and immediately frozen at $-80{ }^{\circ} \mathrm{C}$. cDNAs were synthesized, purified with Ampure XP beads and then amplified with 13 PCR cycles with Advantage 2 Polymerase Mix (50x, Clontech). The PCR products were purified on SPRI AMPure XP beads (Beckman Coulter), and the size distribution was checked on a high-sensitivity DNA chip (Agilent Bioanalyzer). cDNA libraries were prepared with TruSeq Nano DNA kit or Nextera XT DNA (Illumina). For TruSeq Nano libraries, 20-30 ng cDNA was sheared by sonication (parameters adjusted to obtain fragments from 350 to $450 \mathrm{bp}$ ). For Nextera libraries, $1 \mathrm{ng}$ was fragmented by tagmentation. Then cDNA libraries were prepared according to the manufacturer's recommendations. Samples were sequenced on an Illumina HiSeq 2000 at an average of 72.3 million 100 -bp paired-end reads. RNA-seq data have been deposited in the European Nucleotide Archive from EMBL-EBI [38].

\section{Data analysis of the duct transcriptome}

Before mapping, the first 30 bases of each read were trimmed to remove the adapters incorporated by the cDNA synthesis process. Trimmed reads were mapped to the zebrafish genome ( $\mathrm{Zv} 9$, Ensembl genes version 75, ensembl.org) using the Tophat v.2.0.9 software [74]. For the three replicates, the total number of reads for Duct 1 was 40,336,250 (79.9 \% mapped), for Duct 2 was 86,308,531 (82.5 \% mapped), and for Duct 3 was 91,980,011 (67.6 \% mapped).

HT-Seq count was used to estimate the expression level by counting how many reads align to each gene of the annotation (gene set, Ensembl.org) [75]. The expression of 15,888 genes was detected with at least one read in all three replicates.

To describe the set of genes enriched or preferentially expressed in ductal tissue, the ductal transcriptome was compared with acinar and endocrine transcriptomes prepared following the same methodology (composed of alpha, beta, and delta cells, not shown here, manuscript in preparation). The $\mathrm{R}$ package EBSeq was used to call differential expressed genes [76]. Ductal genes were identified based on their posterior probability (adjusted by false discovery rate) of being differentially expressed from the other two cell types. It was found that 3,684 genes were preferentially expressed in the duct transcriptome. Stricter thresholds were applied to identify genes with highly specific ductal expression (see "Results").

\section{Additional files}

Additional file 1: Figure S1. The bacterial artificial chromosome (BAC) reporter line $\operatorname{Tg}(n k \times 6.1:$ GFP) mirrors the expression of the endogenous $n k x 6.1$ gene. A Schematic representation of the -55 to $+95 \mathrm{~kb}$ nkx6.1:eGFP BAC transgene. This BAC includes sequence blocks that are highly conserved amongst vertebrates (black boxes) located from 11 to $77 \mathrm{~kb}$ downstream of the $n k \times 6.1$ gene. By BAC recombineering using galK selection, the eGFP cassette (green box) was introduced into exon 1, replacing the beginning of the $n k x 6.1$ open reading frame (aa 1 to 149). B Epifluorescence microscopy images of the immunodetection of endogenous Nkx6.1 (red) and GFP (green) in Tg(nkx6.1:eGFP) embryos. Lateral views of $48 \mathrm{hpf}$ embryos with the anterior part to the left. $\mathbf{C}$ Confocal projection images of whole mount fluorescent in situ hybridization on a $\operatorname{Tg}(n k \times 6.1: e G F P)$ embryo showing that the gfp transcripts are not expressed in the insulin+ cells at 20 hpf. D Confocal projection images of whole mount fluorescent in situ hybridization on a $\mathrm{Tg}$ (nkx6.1:eGFP) embryo showing that the gfp transcripts are not expressed in the is $11+$ cells at $30 \mathrm{hpf}$. Scale bar $=15 \mu \mathrm{m}$. $P$ pancreas. (PNG $2609 \mathrm{~kb}$ )

Additional file 2: Figure S2. The bacterial artificial chromosome (BAC) reporter line $\mathrm{Tg}$ (ascl1b:eGFP-creER ${ }^{T 2}$ ) mirrors the expression of the endogenous asclib gene. A Schematic representation of the -61 to +89 $\mathrm{kb}$ ascl1b:eGFP-2A-creER ${ }^{T 2}$ BAC transgene. By BAC recombineering using galK selection, the eGFP-2A-creER ${ }^{T 2}$ cassette is introduced into exon 1, replacing the beginning of the asclib open reading frame (aa 1 to 163). B Epifluorescence microscopy images of the immunodetection of GFP in 17-hpf Tg(ascl1b:eGFP-creER ${ }^{T 2}$ ) embryos. C, D Visible WISH showing expression of endogenous ascl1b in a wild-type (WT) embryo (C) and of GFP in Tg(ascl1b:eGFP-creER ${ }^{T 2}$ ) embryos (D) at 15 hpf. Lateral views with the anterior part to the left. $P$ pancreas. (PNG $1068 \mathrm{~kb}$ )

Additional file 3: Figure S3. $n k \times 6.1$ expression is not repressed by Ascl1b. A Schematic representation of wild-type (WT) and mutant Ascl1 bulg-M2C proteins. The basic domain $(b)(+70 /+78)$ is represented by a blue box and the helix loop helix $(H L H)$ domain $(+79 /+123)$ by a green box. The coding region of the mutant Ascl1 $b^{\text {ulg-M2C }}$ protein contains an 11-bp deletion after the aa 107 codon, leading to a frameshift and the production of an aberrant region of 48 aa instead of the second helix, known to be essential for the function of the bHLH proteins. A' Table showing part of the nucleotide and protein sequence of the asclib gene and of the mutated form in the Ascl1 bulg-M2C mutant. B, C WISH showing the drastic reduction of somatostatin expression in the ascl1 $\mathrm{b}^{-/-}$mutant compared to the WT embryo at $30 \mathrm{hpf}$. This phenotype is identical to the one of embryos injected with a translation-blocking morpholino, targeting the translation start site of ascl1b mRNA $[15,77,78]$, suggesting that the mutant Ascl1 $b^{\text {ulg-M2C }}$ is effectively a null mutant. D, E Confocal projection images of Nkx6.1 immunodetection showing equivalent 
number of nkx6.1+ cells in WT and ascl1 b- $^{-1}$ mutants at 16 hpf. All views are ventral with the anterior part to the left. Scale bars $=40 \mu \mathrm{m}$. (PNG $1321 \mathrm{~kb}$ )

Additional file 4: Figure S4. asclib expression is not repressed by Nkx6.1. A Schematic representation of wild-type (WT) and mutant

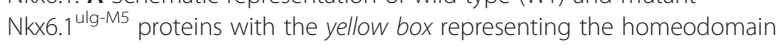
and the red box representing the NK domain. The coding region of the mutant Nkx6.1 $1^{\text {ulg-M5 }}$ protein contains a 7-bp deletion after the aa 141 codon, leading to the apparition of a STOP codon just after the deletion. The black line represents the region of the protein recognized by the Nkx6.1 antibody. A' Table showing part of the nucleotide and protein sequence of the $n k \times 6.1$ gene and of the mutated form in the Nkx6.1 ulg-Ms mutant. B, C Nkx6.1 immunodetection showing the loss of the full-length Nkx6.1 protein in the $n k \times 6.1 \%$ mutant. D, E Glucagon immunodetection showing a drastic reduction of the number of glucagon-expressing cells in the $n k \times 6.1^{-1-}$ mutant compared to the WT embryo at $30 \mathrm{hpf}$. This phenotype is the same as the one of embryos injected with the MO1 translation-blocking morpholino, targeting the translation start site of $n k \times 6.1$ mRNA [18], shown to prevent $n k \times 6.1$ expression efficiently in the neural tube [72]. This strongly suggests that the mutant Nkx6.1 $1^{\text {lg-M5 }}$ is a null mutant. F, G Confocal projection images of fluorescent WISH showing equivalent number of asclib+ cells in WT and $n k \times 6.1^{-1-}$ mutants at $15 \mathrm{hpf}$. All views are ventral with the anterior part to the left. Scale bars $=40 \mu \mathrm{M}$. (PNG $1527 \mathrm{~kb})$

Additional file 5: Figure S5. The initiation of $n k \times 6.1$ expression is independent of Notch signaling. A-D Fluorescent WISH showing a drastic increase in the pancreatic dorsal bud of asclib expression in the mind bomb (mib--) mutants while $n k \times 6.1$ expression is not affected at 13 hpf. E, F Fluorescent WISH showing that the pancreatic expression of $n k \times 6.1$ in the ventral bud is not perturbed at $34 \mathrm{hpf}$ in mib $^{-1}$ mutants. The ventral $(\mathbf{A}, \mathbf{B}, \mathbf{E}, \mathbf{F})$ or lateral $(\mathbf{C}, \mathbf{D})$ views represent confocal projection images with the anterior to the left. Scale bars $=40 \mu \mathrm{M}$. NS nervous system. (PNG $968 \mathrm{~kb}$ )

Additional file 6: Figure S6. All Notch-responsive cells in the adult pancreas are CACs. Notch active cells in $T g$ (Tp 1:VenusPest) adult fish were labeled with anti-GFP to visualize VenusPest (green) and the ductal marker 2F11 (red). VenusPest labeling is detected throughout the exocrine tissue (see asterisks highlighting examples) and where it highlights the CACs together with 2F11. In contrast, ductal structures with intense 2F11 staining do not exhibit any Venus+ (Notch on) cells. Note also the presence of $2 \mathrm{~F} 11$ in endocrine islet cells as previously reported [79]. (PNG 2854 kb)

Additional file 7: Figure S7. Notch-responsive terminal end duct cells/ CACs give rise to other ductal cells. Immunodetection of $\mathrm{H} 2 \mathrm{BmCherry}$ and ductal markers in the pancreas of adult $\operatorname{Tg}(T p 1$ :VenusPest); Tg(Tp1:H2BmCherry) zebrafish. A-C H2BmCherry+ cells (red) in ductular structures labeled with the ductal (ducts and CACs) marker 2F11 (green). A, B separate channels of Fig. 8b-C. B, C Weak H2BmCherry labeling is present near the extremity of a terminal (or intercalated) duct (yellow arrows). The asterisks identify a CAC at the tip of the duct (strong H2BmCherry labeling). D Comparison of $\mathrm{H} 2 \mathrm{BmCherry}$ (red) with endogenous Nkx6.1 (green) showing ductal Nkx6.1+ cells co-expressing H2BmCherry (yellow arrows). (PNG 4967 kb)

Additional file 8: Table S1. Duct-specific genes listed by decreased expression level. We found 293 genes corresponding to the following "specificity" criteria: gene expression level normalized counts > 1000 in duct and $<1000$ in non-ductal (averaged endocrine and acinar) transcriptome and $\geq 16$ fold enrichment in ducts versus non-duct (averaged endocrine and acinar), expressed in $\log _{2}$ ratio ( $\geq 4$ ). Ratio is mean of normalized counts in duct transcriptomes/mean of normalized counts in endocrine and in acinar transcriptomes. Known ductal markers and genes involved in the Notch pathway are highlighted in red. Novel markers and genes involved in the Wht pathway are highlighted in green. (DOCX $26 \mathrm{~kb}$ )

Additional file 9: Figure S8. Ductal expression of id2a during zebrafish development. Whole mount fluorescent in situ hybridization showing idza (green) and the ductal marker sox9b (red) in a 3-dpf larva. The expression of both genes overlaps as highlighted by yellow labeling. In addition to expression in the intrapancreatic ducts (IPD), id2a is also expressed in the intrahepatic (IHD). HPD hepatopancreatic ducts. (PNG $537 \mathrm{~kb}$ )
Additional file 10: Table S2. List of the primers used in this study. The 50 nucleotides added to allow homologous recombination are indicated in capital letters. (DOCX $12 \mathrm{~kb}$ )

\section{Abbreviations}

4OHT: 4-hydroxytamoxifen; aa: amino acid; ARP: atonal related protein; ASCL: achaete-scute like; BAC: bacterial artificial chromosome; bHLH: basic helix-loop-helix; bp: base pair; CAC: centroacinar/terminal end duct cell; creER $^{T 2}$ : $4 \mathrm{OHT}$ inducible Cre-recombinase; DMSO: dimethyl sulfoxide; dpf: days post fertilization; DOG: two-deoxy-D-galactose; dpt: days post treatment; EdU: 5-ethynyl-2'-deoxyuridine; eGFP: enhanced green fluorescent protein; EPD: extra-pancreatic duct; FACS: fluorescence-activated cell sorting; Gcg: glucagon; GFP: green fluorescent protein; hpf: hours post fertilization; IHC: immunohistochemistry; Ins: insulin; IPD: intra-pancreatic duct; kb: kilobyte; MTZ: metronidazole; NICD: Notch intracellular domain; NTR: nitroreductase enzyme; PCNA: proliferating cell nuclear antigen; PCR: polymerase chain reaction; PNC: pancreatic Notch-responsive cell; rec: recombinant; Sst: somatostatin; VenusPest: Venus fluorescent protein; WISH: whole-mount in situ hybridization; WT: wild type.

\section{Competing interests}

The authors declare that they have no competing interests.

\section{Authors' contributions}

APG and DB participated in the design of the study, generated the data and participated in their interpretation, and in the drafting of the manuscript. ETS carried out the RNA-seq analyses. LCF initiated the study on asclib (design and experiments). WB participated in the generation of experimental data. BP participated in the design of the study and in data interpretation, and helped draft the manuscript. MLV and IM conceived the study, participated in the interpretation of the data, and wrote the manuscript. All authors read and approved the final manuscript.

\section{Acknowledgments}

We are grateful to Christian Mosiman for advice on tamoxifen treatment and Marielle Lebrun and Alain Vanderplasschen for advice on galk recombineering. We thank Koichi Kawakami for the itol2 cassette [25]. We are very grateful to Didier Stainier and Nicolay Ninov for the transgenic lines Tg(Tp1:VenusPest) and Tg(Tp1:H2BmCherry) [16], Francesco Argenton for Tg(Tp1glob:eGFP) [12], Carole Wilson (UCL fish facility, UK) for Tg(ubi:Switch) [28], Geoffrey Burns for Tg(ubi:loxP-AmCyan-IoxP-ZsYellow), and Jean-Claude TWIZERE for Tg(hsp70:Gal4) and Tg(UAS:myc-notchia-intra) [33]. We thank the following technical platforms: GIGA-Zebrafish (M Winandy and H Pendeville), GIGA-Cell Imaging and Flow Cytometry platform (S Ormenese and S Raafat), GIGA-Genotranscriptomic (B Hennuy, W Coppieters, and L Karim), and GIGAImmunohistochemistry (C Humblet and E Dortu).

APG and DB were supported by FRIA (Fonds pour la Formation à la Recherche dans l'Industrie et dans l'Agriculture) and the Léon Fredericq fund, ETS by WBI (Wallonie-Bruxelles International) and the Léon Frederica fund, and WB by the Belgian State's Interuniversity Attraction Poles Program (SSTC, PAI). BP, IM, and MLV are Chercheur qualifié FNRS (Fonds National pour la Recherche Scientifique). This work was funded by the FNRS-FRS, the Belgian State's Interuniversity Attraction Poles Program (SSTC, PAI), and the Fonds Speciaux from the ULg (University of Liège).

\section{Received: 21 July 2015 Accepted: 18 August 2015} Published online: 02 September 2015

\section{References}

1. Pisharath H, Rhee JM, Swanson MA, Leach SD, Parsons MJ. Targeted ablation of beta cells in the embryonic zebrafish pancreas using $E$. coli nitroreductase. Mech Dev. 2007;124:218-29.

2. Moss JB, Koustubhan P, Greenman M, Parsons MJ, Walter I, Moss LG. Regeneration of the pancreas in adult zebrafish. Diabetes. 2009;58:1844-51.

3. Curado S, Anderson RM, Jungblut B, Mumm J, Schroeter E, Stainier DY. Conditional targeted cell ablation in zebrafish: a new tool for regeneration studies. Dev Dyn. 2007;236:1025-35

4. Field HA, Dong PD, Beis D, Stainier DY. Formation of the digestive system in zebrafish. II. Pancreas morphogenesis. Dev Biol. 2003;261:197-208. 
5. Kumar M, Melton D. Pancreas specification: a budding question. Curr Opin Genet Dev. 2003;13:401-7.

6. Biemar F, Argenton F, Schmidtke R, Epperlein S, Peers B, Driever W. Pancreas development in zebrafish: early dispersed appearance of endocrine hormone expressing cells and their convergence to form the definitive islet. Dev Biol. 2001;230:189-203.

7. Wang Y, Rovira M, Yusuff S, Parsons MJ. Genetic inducible fate mapping in larval zebrafish reveals origins of adult insulin-producing beta-cells. Development. 2011;138:609-17.

8. Hesselson D, Anderson RM, Beinat M, Stainier DY. Distinct populations of quiescent and proliferative pancreatic beta-cells identified by HOTcre mediated labeling. Proc Natl Acad Sci U S A. 2009;106:14896-901.

9. Kimmel RA, Onder L, Wilfinger A, Ellertsdottir E, Meyer D. Requirement for Pdx1 in specification of latent endocrine progenitors in zebrafish. BMC Biol. 2011;9:75.

10. Chung WS, Andersson O, Row R, Kimelman D, Stainier DY. Suppression of Alk8-mediated Bmp signaling cell-autonomously induces pancreatic beta-cells in zebrafish. Proc Natl Acad Sci U S A. 2010;107:1142-7.

11. Dong PD, Munson CA, Norton W, Crosnier C, Pan X, Gong Z, et al. Fgf10 regulates hepatopancreatic ductal system patterning and differentiation. Nat Genet. 2007;39:397-402.

12. Parsons MJ, Pisharath $\mathrm{H}$, Yusuff S, Moore JC, Siekmann AF, Lawson N, et al. Notch-responsive cells initiate the secondary transition in larval zebrafish pancreas. Mech Dev. 2009:126:898-912.

13. Afelik $\mathrm{S}$, Jensen J. Notch signaling in the pancreas: patterning and cell fate specification. Wiley Interdiscip Rev Dev Biol. 2013;2:531-44.

14. Apelqvist A, Li H, Sommer L, Beatus P, Anderson DJ, Honjo T, et al. Notch signalling controls pancreatic cell differentiation. Nature. 1999:400:877-81.

15. Flasse LC, Pirson JL, Stern DG, Von Berg V, Manfroid I, Peers B, et al. Ascl1 b and Neurod1, instead of Neurog3, control pancreatic endocrine cell fate in zebrafish. BMC Biol. 2013;11:78.

16. Ninov N, Borius M, Stainier DY. Different levels of Notch signaling regulate quiescence, renewal and differentiation in pancreatic endocrine progenitors. Development. 2012;139:1557-67.

17. Zhou Q, Law AC, Rajagopal J, Anderson WJ, Gray PA, Melton DA. A multipotent progenitor domain guides pancreatic organogenesis. Dev Cell. 2007;13:103-14

18. Binot AC, Manfroid I, Flasse L, Winandy M, Motte P, Martial JA, et al. Nkx6.1 and $n k \times 6.2$ regulate alpha- and beta-cell formation in zebrafish by acting on pancreatic endocrine progenitor cells. Dev Biol. 2010;340:397-407.

19. Schaffer $A E$, Freude KK, Nelson SB, Sander M. Nkx6 transcription factors and Ptf1a function as antagonistic lineage determinants in multipotent pancreatic progenitors. Dev Cell. 2010:18:1022-9.

20. Hesselson D, Anderson RM, Stainier DY. Suppression of Ptf1a activity induces acinar-to-endocrine conversion. Curr Biol. 2011;21:712-17.

21. Dong PD, Provost E, Leach SD, Stainier DY. Graded levels of Ptf1a differentially regulate endocrine and exocrine fates in the developing pancreas. Genes Dev. 2008;22:1445-50.

22. Manfroid I, Ghaye A, Naye F, Detry N, Palm S, Pan L, et al. Zebrafish sox9b is crucial for hepatopancreatic duct development and pancreatic endocrine cell regeneration. Dev Biol. 2012;366:268-78.

23. Sander M, Sussel L, Conners J, Scheel D, Kalamaras J, Dela Cruz F, et al. Homeobox gene Nkx6.1 lies downstream of Nkx2.2 in the major pathway of beta-cell formation in the pancreas. Development. 2000;127:5533-40.

24. Suster ML, Sumiyama K, Kawakami K. Transposon-mediated BAC transgenesis in zebrafish and mice. BMC Genomics. 2009:10:477.

25. Suster ML, Abe G, Schouw A, Kawakami K. Transposon-mediated BAC transgenesis in zebrafish. Nat Protoc. 2011;6:1998-2021.

26. Corish $P$, Tyler-Smith C. Attenuation of green fluorescent protein half-life in mammalian cells. Protein Eng. 1999;12:1035-40.

27. Zhou Y, Cashman TJ, Nevis KR, Obregon P, Carney SA, Liu Y, et al. Latent TGF-beta binding protein 3 identifies a second heart field in zebrafish. Nature. 2011;474:645-8.

28. Mosimann C, Kaufman CK, Li P, Pugach EK, Tamplin OJ, Zon LI. Ubiquitous transgene expression and Cre-based recombination driven by the ubiquitin promoter in zebrafish. Development. 2011;138:169-77.

29. Chung WS, Shin CH, Stainier DY. Bmp2 signaling regulates the hepatic versus pancreatic fate decision. Dev Cell. 2008;15:738-48.

30. Chung WS, Stainier DY. Intra-endodermal interactions are required for pancreatic beta cell induction. Dev Cell. 2008;14:582-93.

31. Hwang WY, Fu Y, Reyon D, Maeder ML, Tsai SQ, Sander JD, et al. Efficient genome editing in zebrafish using a CRISPR-Cas system. Nat Biotechnol. 2013;31:227-9.
32. Itoh $\mathrm{M}$, Kim CH, Palardy $\mathrm{G}$, Oda T, Jiang $\mathrm{Y}$, Maust $\mathrm{D}$, et al. Mind bomb is a ubiquitin ligase that is essential for efficient activation of Notch signaling by Delta. Dev Cell. 2003;4:67-82.

33. Scheer N, Groth A, Hans S, Campos-Ortega JA. An instructive function for Notch in promoting gliogenesis in the zebrafish retina. Development. 2001;128:1099-107.

34. Lorent K, Moore JC, Siekmann AF, Lawson N, Pack M. Reiterative use of the notch signal during zebrafish intrahepatic biliary development. Dev Dyn. 2010;239:855-64

35. Ninov N, Hesselson D, Gut P, Zhou A, Fidelin K, Stainier DY. Metabolic regulation of cellular plasticity in the pancreas. Curr Biol. 2013;23:1242-50.

36. Matsuda H, Parsons MJ, Leach SD. Aldh1-expressing endocrine progenitor cells regulate secondary islet formation in larval zebrafish pancreas. PLoS One. 2013;8, e74350.

37. Delaspre F, Beer RL, Rovira M, Huang W, Wang G, Gee S, et al. Centroacinar cells are progenitors that contribute to endocrine pancreas regeneration. Diabetes. 2015. Epub ahead of print

38. European Nucleotide Archive. RNAseq from mature ductal cells from nkx6.1:GFP zebrafish lines. http://www.ebi.ac.uk/ena/data/view/PRJEB10137

39. Shih HP, Kopp JL, Sandhu M, Dubois CL, Seymour PA, Grapin-Botton A, et al. A Notch-dependent molecular circuitry initiates pancreatic endocrine and ductal cell differentiation. Development. 2012;139:2488-99.

40. Zecchin E, Mavropoulos A, Devos N, Filippi A, Tiso N, Meyer D, et al. Evolutionary conserved role of ptf1a in the specification of exocrine pancreatic fates. Dev Biol. 2004:268:174-84.

41. Briscoe J, Pierani A, Jessell TM, Ericson J. A homeodomain protein code specifies progenitor cell identity and neuronal fate in the ventral neural tube. Cell. 2000;101:435-45.

42. Olguin HC, Yang Z, Tapscott SJ, Olwin BB. Reciprocal inhibition between Pax7 and muscle regulatory factors modulates myogenic cell fate determination. J Cell Biol. 2007;177:769-79.

43. Laslo P, Spooner CJ, Warmflash A, Lancki DW, Lee HJ, Sciammas R, et al. Multilineage transcriptional priming and determination of alternate hematopoietic cell fates. Cell. 2006;126:755-66.

44. Afelik S, Qu X, Hasrouni E, Bukys MA, Deering T, Nieuwoudt S, et al. Notch-mediated patterning and cell fate allocation of pancreatic progenitor cells. Development. 2012;139:1744-53.

45. Nelson SB, Schaffer AE, Sander M. The transcription factors Nkx6.1 and Nkx6.2 possess equivalent activities in promoting beta-cell fate specification in Pdx1+ pancreatic progenitor cells. Development. 2007;134:2491-500.

46. Rovira M, Scott SG, Liss AS, Jensen J, Thayer SP, Leach SD. Isolation and characterization of centroacinar/terminal ductal progenitor cells in adult mouse pancreas. Proc Natl Acad Sci U S A. 2010;107:75-80.

47. Kopinke D, Brailsford M, Shea JE, Leavitt R, Scaife CL, Murtaugh LC. Lineage tracing reveals the dynamic contribution of Hes1+ cells to the developing and adult pancreas. Development. 2011;138:431-41.

48. Zhou Q, Brown J, Kanarek A, Rajagopal J, Melton DA. In vivo reprogramming of adult pancreatic exocrine cells to beta-cells. Nature. 2008:455:627-32

49. Ferber S, Halkin A, Cohen H, Ber I, Einav Y, Goldberg I, et al. Pancreatic and duodenal homeobox gene 1 induces expression of insulin genes in liver and ameliorates streptozotocin-induced hyperglycemia. Nat Med. 2000;6:568-72.

50. Shternhall-Ron K, Quintana FJ, Perl S, Meivar-Levy I, Barshack I, Cohen IR, et al. Ectopic PDX-1 expression in liver ameliorates type 1 diabetes. J Autoimmun. 2007:28:134-42.

51. Ber I, Shternhall K, Perl S, Ohanuna Z, Goldberg I, Barshack I, et al. Functional, persistent, and extended liver to pancreas transdifferentiation. Biol Chem. 2003:278:31950-7.

52. Miyashita K, Miyatsuka T, Matsuoka TA, Sasaki S, Takebe S, Yasuda T, et al. Sequential introduction and dosage balance of defined transcription factors affect reprogramming efficiency from pancreatic duct cells into insulinproducing cells. Biochem Biophys Res Commun. 2014;444:514-9.

53. Gefen-Halevi S, Rachmut IH, Molakandov K, Berneman D, Mor E, Meivar-Levy I, et al. NKX6.1 promotes PDX-1-induced liver to pancreatic beta-cells reprogramming. Cell Reprogram. 2010;12:655-64.

54. Ye L, Robertson MA, Hesselson D, Stainier DY, Anderson RM. Glucagon is essential for alpha cell transdifferentiation and beta cell neogenesis. Development. 2015;142:1407-17.

55. Miyamoto Y, Maitra A, Ghosh B, Zechner U, Argani P, lacobuzio-Donahue CA et al. Notch mediates TGF alpha-induced changes in epithelial differentiation during pancreatic tumorigenesis. Cancer Cell. 2003;3:565-76. 
56. Strobel O. In vivo lineage tracing defines the role of acinar-to-ductal transdifferentiation in inflammatory ductal metaplasia. Gastroenterology. 2007;133:1999-2009.

57. Stanger BZ, Stiles B, Lauwers GY, Bardeesy N, Mendoza M, Wang Y, et al. Pten constrains centroacinar cell expansion and malignant transformation in the pancreas. Cancer Cell. 2005;8:185-95.

58. Hua H, Zhang YQ, Dabernat S, Kritzik M, Dietz D, Sterling L, et al. BMP4 regulates pancreatic progenitor cell expansion through Id2. J Biol Chem. 2006;281:13574-80

59. Collu GM, Hidalgo-Sastre A, Brennan K. Wnt-Notch signalling crosstalk in development and disease. Cell Mol Life Sci. 2014;71:3553-67.

60. Baumgartner BK, Cash G, Hansen H, Ostler S, Murtaugh LC. Distinc requirements for beta-catenin in pancreatic epithelial growth and patterning. Dev Biol. 2014;391:89-98.

61. Westerfield M. The zebrafish book: a guide for the laboratory use of zebrafish (Danio rerio). 3rd ed. Eugene, OR: University of Oregon Press; 1995.

62. Kimmel CB, Ballard WW, Kimmel SR, Ullmann B, Schilling TF. Stages of embryonic development of the zebrafish. Dev Dyn. 1995;203:253-310.

63. Haddon C, Jiang YJ, Smithers L, Lewis J. Delta-Notch signalling and the patterning of sensory cell differentiation in the zebrafish ear: evidence from the mind bomb mutant. Development. 1998;125:4637-44.

64. Warming S, Costantino N, Court DL, Jenkins NA, Copeland NG. Simple and highly efficient BAC recombineering using galK selection. Nucleic Acids Res. 2005;33, e36.

65. Bussmann J, Schulte-Merker S. Rapid BAC selection for tol2-mediated transgenesis in zebrafish. Development. 2011;138:4327-32.

66. Jao LE, Wente SR, Chen W. Efficient multiplex biallelic zebrafish genome editing using a CRISPR nuclease system. Proc Natl Acad Sci U S A. 2013;110:13904-9.

67. ZiFiT Targeter Version 4.2. http://zifit.partners.org/ZiFiT/

68. Ota S, Hisano Y, Muraki M, Hoshijima K, Dahlem TJ, Grunwald DJ, et al. Efficient identification of TALEN-mediated genome modifications using heteroduplex mobility assays. Genes Cells. 2013;18:450-8.

69. Mavropoulos A, Devos N, Biemar F, Zecchin E, Argenton F, Edlund H, et al. sox $4 b$ is a key player of pancreatic alpha cell differentiation in zebrafish. Dev Biol. 2005;285:211-23.

70. Hauptmann G, Gerster T. Two-color whole-mount in situ hybridization to vertebrate and Drosophila embryos. Trends Genet. 1994;10:266.

71. Allende ML, Weinberg ES. The expression pattern of two zebrafish achaete-scute homolog (ash) genes is altered in the embryonic brain of the cyclops mutant. Dev Biol. 1994;166:509-30.

72. Cheesman SE, Layden MJ, Von Ohlen T, Doe CQ, Eisen JS. Zebrafish and fly Nkx6 proteins have similar CNS expression patterns and regulate motoneuron formation. Development. 2004;131:5221-32.

73. Argenton F, Zecchin E, Bortolussi M. Early appearance of pancreatic hormone-expressing cells in the zebrafish embryo. Mech Dev. 1999;87:217-21.

74. Trapnell C, Roberts A, Goff L, Pertea G, Kim D, Kelley DR, et al. Differential gene and transcript expression analysis of RNA-seq experiments with TopHat and Cufflinks. Nat Protoc. 2012;7:562-78.

75. Anders S, Pyl PT, Huber W. HTSeq-a Python framework to work with high-throughput sequencing data. Bioinformatics. 2014;31:166-9.

76. Leng N, Dawson JA, Thomson JA, Ruotti V, Rissman Al, Smits BM, et al. EBSeq: an empirical Bayes hierarchical model for inference in RNA-seq experiments. Bioinformatics. 2013;29:1035-43.

77. Nikolaou N, Watanabe-Asaka T, Gerety S, Distel M, Koster RW, Wilkinson DG. Lunatic fringe promotes the lateral inhibition of neurogenesis. Development. 2009;136:2523-33.

78. Amoyel M, Cheng YC, Jiang YJ, Wilkinson DG. Wnt1 regulates neurogenesis and mediates lateral inhibition of boundary cell specification in the zebrafish hindbrain. Development. 2005;132:775-85.

79. Zhang D, Golubkov VS, Han W, Correa RG, Zhou Y, Lee S, et al. Identification of Annexin A4 as a hepatopancreas factor involved in liver cell survival. Dev Biol. 2014;395:96-110.

\section{Submit your next manuscript to BioMed Central and take full advantage of:}

- Convenient online submission

- Thorough peer review

- No space constraints or color figure charges

- Immediate publication on acceptance

- Inclusion in PubMed, CAS, Scopus and Google Scholar

- Research which is freely available for redistribution

Submit your manuscript at www.biomedcentral.com/submit 